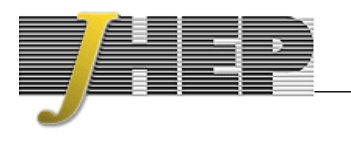

\title{
The tensor hierarchy of 8-dimensional field theories
}

\author{
Óscar Lasso Andino and Tomás Ortín \\ Instituto de Física Teórica UAM/CSIC, \\ C/ Nicolás Cabrera, 13-15, C.U. Cantoblanco, E-28049 Madrid, Spain \\ E-mail: oscar.lasso@estudiante.uam.es, Tomas.Ortin@csic.es
}

ABSTRACT: We construct the tensor hierarchy of generic, bosonic, 8-dimensional field theories. We first study the form of the most general 8-dimensional bosonic theory with Abelian gauge symmetries only and no massive deformations. This study determines the tensors that occur in the Chern-Simons terms of the (electric and magnetic) field strengths and the action for the electric fields, which we determine. Having constructed the most general Abelian theory we study the most general gaugings of its global symmetries and the possible massive deformations using the embedding tensor formalism, constructing the complete tensor hierarchy using the Bianchi identities. We find the explicit form of all the field strengths of the gauged theory up to the 6-forms. Finally, we find the equations of motion comparing the Noether identities with the identities satisfied by the Bianchi identities themselves. We find that some equations of motion are not simply the Bianchi identities of the dual fields, but combinations of them.

KEYwords: Gauge Symmetry, Supersymmetry and Duality

ARXIV EPRINT: 1605.05882 


\section{Contents}

1 Ungauged $d=8$ theories $\quad 4$

2 Abelian, massless deformations $\quad 6$

$\begin{array}{lll}2.1 & \text { The 6-form potentials and their 7-form field strengths } & 11\end{array}$

3 Non-Abelian and massive deformations: the tensor hierarchy 13

$\begin{array}{lll}3.1 & 2 \text {-form field strengths } & 15\end{array}$

$\begin{array}{lll}3.2 & 3 \text {-form field strengths } & 16\end{array}$

$\begin{array}{ll}3.3 & 4 \text {-form field strengths } \\ & 17\end{array}$

$\begin{array}{lll}3.4 & 5 \text {-form field strengths } & 18\end{array}$

$\begin{array}{lll}3.5 & 6 \text {-form field strengths } & 18\end{array}$

$\begin{array}{lll}3.6 & \text { 7-form field strengths } & 19\end{array}$

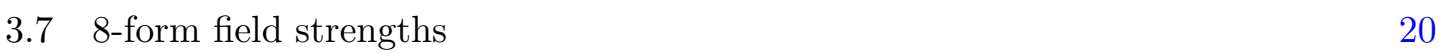

$\begin{array}{ll}3.8 & 9 \text {-form field strengths }\end{array}$

4 Gauge-invariant action for the 1-, 2- and 3-forms 23

5 Conclusions $\quad 27$

A Summary of relations for the generic ungauged, massless Abelian $d=8$ theory

A.1 Field strengths 28

$\begin{array}{lll}\text { A.2 Bianchi identities } & 28\end{array}$

$\begin{array}{lll}\text { A.3 Duality relations } & 28\end{array}$

B Summary of relations for the gauged theory 29

$\begin{array}{lll}\text { B.1 Field strengths } & 29\end{array}$

$\begin{array}{lll}\text { B.2 Bianchi identities } & 29\end{array}$

B.3 Identities of Bianchi identities 30

\section{Introduction}

Over the last years, a great effort has been made to explore the most general field theories. This exploration has been motivated by two main reasons. First of all there is the need to search for viable candidates to describe the fundamental interactions known to us (specially gravity) and the universe at the cosmological scale, solving the theoretical problems encountered by the theories available today. The second reason is the desire to map the space of possible theories and the different relations and dualities existing between them. 
In the String Theory context, the landscape of $\mathcal{N}=1, d=4$ vacua has focused most of the attention, but more general compactifications have also been studied. At the level of the effective field theories the exploration has been carried out within the space of supergravity theories. Most ungauged supergravity theories (excluding those of higher order in curvature) and some of the gauged ones have been constructed in the past century [1], but the space of possible gaugings and massive deformations (related to fluxes, symmetry enhancements etc. in String theory) has started to be studied in a systematic way more recently with the introduction of the embedding-tensor formalism in refs. [2-4]. The formalism was developed in the context of the study of the gauging of $\mathcal{N}=8, d=4$ supergravity in refs. [5, 6], but it has later been used in theories with less supersymmetry in different dimensions. ${ }^{1}$

The embedding-tensor formalism comes with a bonus: the tensor hierarchy $[6,8-11]$. Using electric and magnetic vector fields in $d=4$ dimensions as gauge fields requires the introduction of 2 -form-potentials in the theory, which would be dual to the scalars. In $d=6$ dimensions certain gaugings require the introduction of magnetic 2 -form and 3 -form potentials [12]. But the addition of higher-rank potentials does not stop there: as a general rule, the construction of gauge-invariant field strengths for the new $p$-form fields requires the introduction of $(p+1)$-form fields with Stückelberg couplings. This leads to a tensor hierarchy that includes all the electric and magnetic fields of the theory and opens up the systematic construction of gauged theories: construct the hierarchy using gauge invariance as a principle expressed through the Bianchi identities and find the equations of motion by using the duality relations between electric and magnetic fields of ranks $p$ and $d-p-2$.

This approach has been used in refs. $[10,13]$ to construct the most general 4-, 5and 6-dimensional field theories ${ }^{2}$ with gauge invariance with at most two derivatives. In this paper we want to consider the 8-dimensional case and construct the most general 8dimensional field theory with gauge invariance and of second order in derivatives in the action: tensor hierarchy, Bianchi identities, field strengths, duality relations and action. ${ }^{3}$

One of our main motivations for considering this problem (besides the construction of the most general 8-dimensional gauge theories) is to simplify and systematize the construction of maximal 8-dimensional supergravities with $\mathrm{SO}(3)$ gaugings. All these theories will be equivalent from an 8-dimensional point of view, because, as shown in refs. $[15,16]$ and unlike the $\mathrm{SO}(8)$-gauged $\mathcal{N}=8, d=4$ supergravities constructed in [17], they are all related by $\mathrm{SL}(2, \mathbb{R})$ duality transformations that can be understood as a different changes of variables. ${ }^{4}$ Thus, the simplest mechanical procedure to obtain them from 11-dimensional supergravity would be to perform the standard Scherk-Schwarz reduction [18] that gives an

\footnotetext{
${ }^{1}$ See, for instance, chapter 2 in ref. [7], which contains a pedagogical introduction to the formalism and references.

${ }^{2}$ Not only supergravities, since the use of the embedding-tensor formalism is not restricted to supergravity theories.

${ }^{3}$ The tensor hierarchy of maximal 8-dimensional supergravity has been constructed in ref. [14] in the context if exceptional field theory.

${ }^{4}$ These changes of variables are non-local in terms of the fundamental (electric) fields of the theory although they are purely local in terms of the fields that occur in the tensor hierarchy (both electric and magnetic). Thus, from the standard point of view, since they include electric-magnetic duality rotations, they are non-local transformations of the fields.
} 
8-dimensional $\mathrm{SO}(3)$-gauged maximal supergravity in which the 3 Kaluza-Klein vectors play the role of gauge fields $[19]^{5}$ and then perform the $\mathrm{SL}(2, \mathbb{R})$ duality transformations mentioned above. This can be, technically, quite complicated as electric-magnetic duality transformations are involved. The Kaluza-Klein triplet of vector fields are the first component of a SL $(2, \mathbb{R})$ doublet and, after the duality transformations, the gauge fields are no longer the first component of that doublet, but a general linear combination of the first and the second.

The 8-dimensional SL $(2, \mathbb{R})$ duality transformations have no clear 11-dimensional counterpart, though, and the $\mathrm{SO}(3)$-gauged maximal supergravities in which the triplet of gauge fields are not the first component of the $\mathrm{SL}(2, \mathbb{R})$ doublet obtained in this way cannot be uplifted to 11 dimenisions: the only way to uplift an 8-dimensional solution of these theories would be to undo first the $\mathrm{SL}(2, \mathbb{R})$ rotation converting the solution in a solution of the Salam-Sezgin theory. In other words: there is no known way of dimensional reduce 11-dimensional supergravity to obtain directly any of the $\mathrm{SL}(2, \mathbb{R})$-rotated $\mathrm{SO}(3)$-gauged 8-dimensional maximal supergravities. This makes all these theories, which are equivalent from the 8-dimensional point of view, interesting to obtain explicitly and the general results that we are going to obtain here will allow us to do so in a systematic and rather painless way.

There is one possible exception to the statement that only the Salam-Sezgin theory can be obtained directly from 11-dimensional supergravity, though: the $\mathrm{SO}$ (3)-gauged 8dimensional maximal supergravity in which the triplet of gauge fields are the second component of the $\operatorname{SL}(2, \mathbb{R})$ doublet (they are, precisely, those coming from the reduction of the 11-dimensional 3-form) was obtained in ref. [22] by dimensional reduction of a non-covariant deformation of 11-dimensional supergravity proposed in ref. [23, 24]. ${ }^{6}$ Since this theory was obtained through a very non-standard procedure, we think it is important to check that this procedure yields the correct result. Being able to construct all the $\mathrm{SO}(3)$-gauged 8dimensional maximal supergravities in a systematic way will also allow us to do so as well. One may, then, perhaps, find more general non-covariant deformations of 11-dimensional supergravity leading to the rest of $\mathrm{SO}(3)$-gauged 8-dimensional maximal supergravities.

The problems involving the $\mathrm{SO}(3)$-gauged 8-dimensional maximal supergravities and their possible 11-dimensional origin will be addressed in a forthcoming publication [26]. In this paper we want to consider the general deformations (gaugings and massive transformations) of generic 8-dimensional theories, which is an interesting problem by itself whose solution will provide us with the most general theories with gauge symmetry in 8 dimensions up to two derivatives.

The construction of the most general 8-dimensional theory with gauge symmetry and at most two derivatives, and this paper, are organized as follows: first, in section 1, we study the structure and symmetries (including electric-magnetic dualities of the 3 -form po-

\footnotetext{
${ }^{5}$ Other, more general, gaugings can be obtained via Scherk-Schwarz reduction [20, 21], but it is always the Kaluza-Klein vectors that play the role of gauge fields.

${ }^{6}$ Many gauged supergravities whose 11-dimensional origin is unkonown or, in more modern parlance, they contain non-geometrical fluxes (like Roman's 10-dimensional massive supergravity or alternative, inequivalent gaugings of other theories) can be obtained systematically from this non-covariant deformation of 11-dimensional supergravity [25], which seems to encode many of these non-geometrical fluxes.
} 
tentials) of generic (up to second order in derivatives) 8-dimensional theories with Abelian gauge symmetry and no Chern-Simons terms.

In section 2, we consider Abelian, massless deformations of those theories, which consist, essentially, in the introduction via some constant " $d$-tensors" of Chern-Simons terms in the field strengths and action. The new intereactions are required to preserve the Abelian gauge symmetries and, formally, the symplectic structure of the electric-magnetic duality transformations of the 3 -form potentials. We determine explicitly the form of all the electric and magnetic field strengths up to the 7 -form field strengths, and give the gauge-invariant action in terms of the electric potentials. This will be our starting point for the next stage.

In section 3 we consider the most general gauging and massive deformations (Stückelberg couplings) of the Abelian theory constructed in the previous section using the embedding-tensor formalism. We proceed as in refs. [7, 13], finding Bianchi identities for field strengths from the identities satisfied by the Bianchi identities of the lower-rank field strengths and, then, solving them. We have found the Bianchi identities satisfied by all the field strengths and we have managed to find the explicit form of the field strengths up to the 6 -form.

In this approach, the " $d$-tensors" that define the Chern-Simons terms will be treated in a different way as in ref. [13]: they will not be treated as deformations of the theory to be gauged, but as part of its definition. Therefore, we will not associate to them any dual 7 -form potentials.

In section 4 we study the construction of an action for the theory. The equations of motion are related to the Bianchi identities by the duality relations between electric and magnetic field strengths, but, at least in this case, they are not directly equal to them. In general they can be combinations of the Bianchi identities. To find the right combinations we derive the Noether identities that the off-shell equations of motion of these theories should satisfy as a consistency condition that follows from gauge invariance. Then, we compare those Noether identities with the identities satisfied by the Bianchi identities. Once the equations of motion have been determined in this way, we proceed to the construction of the action, which we achieve up to terms that only contain 1-forms and their derivatives, whose form is too complicated.

Section 5 contains our conclusions and the main formulae (field strengths, Bianchi identities etc.) of the ungauged and gauged theories are collected in the appendices to simplify their use.

\section{Ungauged $d=8$ theories}

In this section we are going to consider the construction of generic (bosonic) $d=8$ theories coupled to gravity containing terms of second order or lower in derivatives of any given field. ${ }^{7}$ The field content of a generic $d=8$ theory are the metric $g_{\mu \nu}$, scalar fields $\phi^{x}$, 1-form fields $A^{I}=A^{I}{ }_{\mu} d x^{\mu}, 2$-form fields $B_{m}=\frac{1}{2} B_{m \mu \nu} d x^{\mu} \wedge d x^{\nu}$ and 3-form fields $C^{a}=$ $\frac{1}{3 !} C^{a}{ }_{\mu \nu \rho} d x^{\mu} \wedge d x^{\nu} \wedge d x^{\rho}$. For the moment, we place no restrictions on the range of the

\footnotetext{
${ }^{7}$ The Chern-Simons (CS) terms may have terms with more than two derivatives, but they do not act on the same field.
} 
indices labeling these fields nor on the symmetry groups that may act on them leaving the theory invariant.

We are going to start by the simplest theory one can construct with these fields to later gauge it and deform it in different ways.

The simplest field strengths one can construct for these fields are their exterior derivatives:

$$
F^{I} \equiv d A^{I}, \quad H_{m} \equiv d B_{m}, \quad G^{a} \equiv d C^{a} .
$$

They are invariant under the gauge transformations

$$
\delta_{\sigma} A^{I}=d \sigma^{I}, \quad \delta_{\sigma} B_{m}=d \sigma_{m}, \quad \delta_{\sigma} C^{a}=d \sigma^{a},
$$

where the local parameters $\sigma^{I}, \sigma_{m}, \sigma^{a}$ are, respectively, 0-, 1-, and 2-forms.

The most general gauge-invariant action which one can write for these fields is

$$
\begin{aligned}
S=\int\left\{-\star \mathbb{1} R+\frac{1}{2} \mathcal{G}_{x y} d \phi^{x} \wedge \star d \phi^{y}+\frac{1}{2} \mathcal{M}_{I J} F^{I} \wedge \star F^{J}+\frac{1}{2} \mathcal{M}^{m n} H_{m} \wedge \star H_{n}\right. \\
\left.-\frac{1}{2} \Im \mathfrak{m} \mathcal{N}_{a b} G^{a} \wedge \star G^{b}-\frac{1}{2} \Re \mathfrak{e} \mathcal{N}_{a b} G^{a} \wedge G^{b}\right\}
\end{aligned}
$$

where the kinetic matrices $\mathcal{G}_{x y}, \mathcal{M}_{I J}, \mathcal{M}^{m n}, \Im \mathfrak{m} \mathcal{N}_{a b}$ as well as the matrix $\Re \mathfrak{e} \mathcal{N}_{a b}$ are scalardependent. ${ }^{8}$ One could add CS terms to this action, but this possibility will arise naturally in what follows.

The equations of motion of the 3 -forms $C^{a}$ can be written in the form ${ }^{9}$

$$
\frac{\delta S}{\delta C^{a}}=-d \frac{\delta S}{\delta G^{a}}=0, \quad \frac{\delta S}{\delta G^{a}}=R_{a} \equiv-\Re \mathfrak{e} \mathcal{N}_{a b} G^{b}-\Im \mathfrak{m} \mathcal{N}_{a b} \star G^{b} .
$$

These equations can be solved locally by introducing a set of dual 3-forms $C_{a}$ implicitly defined through their field strengths $G_{a}$

$$
R_{a}=G_{a} \equiv d C_{a} .
$$

It is convenient to construct vectors containing the fundamental and dual 3-forms:

$$
\left(C^{i}\right) \equiv\left(\begin{array}{c}
C^{a} \\
C_{a}
\end{array}\right), \quad G^{i} \equiv d C^{i},
$$

so that the equations of motion and the Bianchi identities for the fundamental field strengths take the simple form

$$
d G^{i}=0
$$

In other words: we have traded an equation of motion by a Bianchi identity and a duality relation. In what follows we will do the same for all the fields in the action so

\footnotetext{
${ }^{8}$ If $\Re \mathfrak{e} \mathcal{N}_{a b}$ is constant, then the last term is a total derivative.

${ }^{9}$ The equation of motion of a $p$-form field, $\delta S / \delta \omega^{(p)}$, is an $(8-p)-f o r m$ defined by

$$
\delta S=+\frac{\delta S}{\delta \phi^{x}} \wedge \delta \phi^{x}+\frac{\delta S}{\delta A_{I}} \wedge \delta A^{I}+\frac{\delta S}{\delta B^{m}} \wedge \delta B^{m}+\frac{\delta S}{\delta C^{a}} \wedge \delta C^{a} .
$$
}

With our conventions, when acting on $p$-forms, $\star^{2}=(-1)^{p-1}$. 
that, in the end, we will have only a set of Bianchi identities and a set of duality relations between magnetic and electric fields.

The vector of field strengths $G^{i}$ satisfies the following linear, twisted, self-duality constraint

$$
\star G^{i}=\Omega^{i j} \mathcal{W}_{j k} G^{k},
$$

where

$$
\left(\Omega_{i j}\right)=\left(\Omega^{i j}\right) \equiv\left(\begin{array}{cc}
0 & \mathbb{1} \\
-\mathbb{1} & 0
\end{array}\right),
$$

is the symplectic metric and

$$
\left(\mathcal{W}_{i j}(\mathcal{N})\right) \equiv-\left(\begin{array}{cc}
I_{a b}+R_{a c} I^{c d} R_{d b} & R_{a c} I^{c b} \\
I^{a c} R_{c b} & I^{a b}
\end{array}\right), \quad \Omega \mathcal{W} \Omega^{T}=\mathcal{W}^{-1}
$$

is a symplectic symmetric matrix. ${ }^{10}$ The equations (1.8) are formally invariant under arbitrary $\operatorname{GL}\left(2 n_{3}, \mathbb{R}\right)$ transformations ( $n_{3}$ being the number of fundamental 3-forms) but, just as it happens for 1-forms in $d=4$, the self-duality constraint eq. (1.9) is only preserved by $\operatorname{Sp}\left(2 n_{3}, \mathbb{R}\right)$. As usual, the only $\operatorname{Sp}\left(2 n_{3}, \mathbb{R}\right)$ transformations which are true symmetries of the equations of motion are those associated to the transformations of the scalars which are isometries of $\mathcal{G}_{x y}$ and which also induce linear transformations of the other kinetic matrices. We will discuss this point in more detail later on.

The dualization of the other fields does not lead to any further restrictions.

In what follows we are going to generalize the simple Abelian theory that we have constructed by deforming it, adding new couplings. We will use two guiding principles: preservation of gauge symmetry (even if it needs to be deformed as well) and preservation of the formal symplectic invariance that we have just discussed.

\section{Abelian, massless deformations}

The deformations that we are going to consider in this section consist, essentially, in the introduction of CS terms in the field strengths and in the action. Stückelberg coupling will be considered later. Only the 3- and 4-form field strengths admit these massless Abelian deformations. It is convenient to start by considering this simple modification of $G^{a}:{ }^{11}$

$$
G^{a}=d C^{a}+d^{a}{ }_{I}^{m} F^{I} B_{m},
$$

where $d^{a}{ }^{m}$ is a constant tensor. The gauge transformations need to be deformed accordingly:

$$
\delta_{\sigma} A^{I}=d \sigma^{I}, \quad \delta_{\sigma} B_{m}=d \sigma_{m}, \quad \delta_{\sigma} C^{a}=d \sigma^{a}-d^{a}{ }_{I}^{m} F^{I} \sigma_{m} .
$$

\footnotetext{
${ }^{10}$ Basically the same that occurs in $d=4$ theories, $\mathcal{M}(\mathcal{N})$ see e.g. ref. [27]. We use a slightly different convention for the sake of convenience and $\mathcal{M}(\mathcal{N})=\mathcal{M}(-\mathcal{N})$ due to the unconventional sign on the definition of $G_{a}$.

${ }^{11}$ We will often suppress the wedge product symbols $\wedge$ in order to simplify the expressions that involve differential forms.
} 
The action eq. (1.3) remains gauge-invariant but the formal symplectic invariance is broken: if we do not modify the action, the dual 4-form field strengths are just $G_{a}=d C_{a}$ and $\operatorname{Sp}\left(2 n_{3}, \mathbb{R}\right)$ cannot rotate these into $G^{a}$ in eq. (2.1). Furthermore, the 1-form and 2-form equations of motion do not have a symplectic-invariant form.

This problem can be solved by adding a CS term to the action:

$$
S_{C S}=\int\left\{-d_{a I}^{m} d C^{a} F^{I} B_{m}\right\}
$$

that modifies the equations of motion of the 3 -forms

$$
-d \frac{\delta S}{\delta d C^{a}}=0, \quad \frac{\delta S}{\delta d C^{a}}=R_{a}-d_{a I}^{m} F^{I} B_{m} .
$$

The local solution is now

$$
d C_{a} \equiv R_{a}-d_{a I}^{m} F^{I} B_{m},
$$

and, since $R_{a}$ is gauge-invariant, the dual, gauge-invariant, field strength must be defined by

$$
R_{a}=d C_{a}+d_{a I}^{m} F^{I} B_{m} \equiv G_{a} .
$$

Again, $\left(C^{i}\right)=\left(\begin{array}{c}C^{a} \\ C_{a}\end{array}\right)$ transforms linearly as a symplectic vector if $\left(d^{i} I^{m}\right) \equiv\left(\begin{array}{c}d^{a} I^{m} \\ d_{a I}^{m}\end{array}\right)$ also does. Then, we can define the symplectic vector of 4 -form field strengths

$$
G^{i}=d C^{i}+d_{I}^{i}{ }^{m} F^{I} B_{m},
$$

invariant under the deformed gauge transformations

$$
\delta_{\sigma} A^{I}=d \sigma^{I}, \quad \delta_{\sigma} B_{m}=d \sigma_{m}, \quad \delta_{\sigma} C^{i}=d \sigma^{i}-d^{i}{ }_{I}^{m} F^{I} \sigma_{m} .
$$

However, the deformed gauge transformations do not leave invariant the CS term eq. (2.3). The only solution ${ }^{12}$ is to add another term of the form ${ }^{13}$

$$
S_{C S}=\int\left\{-d_{a I}^{m} d C^{a} F^{I} B_{m}-\frac{1}{2} d_{a I}^{m} d^{a}{ }^{n} F^{I J} B_{m n}\right\}
$$

provided the following constraint holds:

$$
d_{a(I}^{[m} d_{J)}^{a}{ }^{n]}=0, \quad \text { so } \quad d_{i(I}^{m} d_{J)}^{i}=0 .
$$

Observe that we are just using formal symplectic invariance: ${ }^{14}$ the symplectic vector $d_{I}^{i}{ }_{I}^{m}$ is transformed into a different one. Thus, in general, one gets $\operatorname{Sp}\left(2 n_{3}, \mathbb{R}\right)$ multiplets of theories, except when $d_{I}^{i}{ }^{m}$ is a symplectic invariant tensor,${ }^{15}$ which requires, at least,

\footnotetext{
${ }^{12}$ We have not found any other.

${ }^{13}$ We use the compact notation $A^{I J \cdots}=A^{I} A^{J} \cdots, F^{I J \cdots}=F^{I} F^{J} \cdots, B_{m n \ldots}=B_{m} B_{n} \cdots$ etc., where we have suppressed the wedge product symbols.

${ }^{14}$ In other words: we are just saying that these theories, as the 4-dimensional ones, have a natural symplectic structure that should be reflected in the indices of these tensors. The theories may or may not have any symmetries but, if they do, they will be embedded in the symplectic group and the deformation tensors must transform in definite representations of the symplectic group.

${ }^{15}$ The only symplectic-invariant vector is 0 .
} 
one of the indices $I$ or $m$ to be a symplectic index. In most cases the part of the symmetry group of the theory acting on the 3 -forms, while embedded in $\operatorname{Sp}\left(2 n_{3}, \mathbb{R}\right)$, will be a much smaller group and, then, full symplectic invariance of $d_{I}^{i}{ }^{m}$ may not be required.

As a nice check of the formal symplectic invariance of the deformed theory, we can check this invariance on the dual field strengths of the remaining fields, ${ }^{16}$ which is tantamount to checking the invariance of the equations of motion of the fundamental fields.

Using the duality relation $R_{a}=G_{a}$ the equations of motion of the 1-forms can be written in the form

$$
\frac{\delta S}{\delta A^{I}}=-d\left\{\mathcal{M}_{I J} \star F^{J}-d_{i I}{ }^{m} G^{i} B_{m}-\frac{1}{2} d_{i I}{ }^{m} d^{i}{ }_{J}^{n} F^{J} B_{m n}\right\}=0,
$$

and can be solved by identifying all the terms inside the brackets with $d \tilde{A}_{I}$, where $\tilde{A}_{I}$ is a set of 5 -forms. Taking into account gauge invariance, the 6 -form field strengths $\tilde{F}_{I}$ have the following definition, duality relation and Bianchi identities:

$$
\begin{aligned}
\tilde{F}_{I} & \equiv d \tilde{A}_{I}+d_{i I}^{m} G^{i} B_{m}+\frac{1}{2} d_{i I}^{m} d^{i}{ }_{J}^{n} F^{J} B_{m n}, \\
\tilde{F}_{I} & =\mathcal{M}_{I J} \star F^{J} \\
d \tilde{F}_{I} & =d_{i I}{ }^{m} G^{i} H_{m}
\end{aligned}
$$

and the equations of motion are of the 1-forms given by the Bianchi identities of the dual 6 -form field strengths up to duality relations:

$$
\frac{\delta S}{\delta A^{I}}=-\left\{d \tilde{F}_{I}-d_{i I}^{m} G^{i} H_{m}\right\}
$$

Using the duality relation $R_{a}=G_{a}$ and following the same steps for the 2-forms, we find

$$
\begin{aligned}
\tilde{H}^{m} & =d \tilde{B}^{m}+d^{i}{ }_{I}^{m} F^{I} C_{i}, \\
\tilde{H}^{m} & =\mathcal{M}^{m n} \star H_{n}, \\
d \tilde{H}^{m} & =-d_{i I}{ }^{m} G^{i} F^{I},
\end{aligned}
$$

and the equations of motion of the 2-forms are given by the Bianchi identities of the dual 5-form field strengths up to duality relations:

$$
\frac{\delta S}{\delta B_{m}}=-\left\{d \tilde{H}^{m}+d_{i I}^{m} G^{i} F^{I}\right\}
$$

This completes the first Abelian deformation. The second non-trivial deformation of $G^{a}$ that one could consider is the addition of a CS 4-form term $\sim d^{a}{ }_{I J K} A^{I} F^{J} A^{K}$. The gauge transformation of this term is not a total derivative and we cannot make $G^{a}$ gaugeinvariant by deforming the gauge transformation rule of $C^{a}$ only: we must also deform that of $B_{m}$, which, in its turn, induces a deformation of $H_{m}$ by addition of a CS 3-form term.

\footnotetext{
${ }^{16}$ We leave aside the scalars for the moment.
} 
Since the deformation of $H_{m}$ is essentially unique, it is more convenient to start from this side and redefine

$$
H_{m}=d B_{m}-d_{m I J} F^{I} A^{J}
$$

where $d_{m I J}=d_{m J I}{ }^{17}$ which is invariant under the gauge transformations

$$
\delta_{\sigma} A^{I}=d \sigma^{I}, \quad \delta_{\sigma} B_{m}=d \sigma_{m}+d_{m I J} F^{I} \sigma^{J},
$$

and satisfies the Bianchi identities

$$
d H_{m}=-d_{m I J} F^{I J}
$$

Under these gauge transformations and a generic $\delta_{\sigma} C^{a}$

$$
\delta_{\sigma} G^{a}=d\left(\delta_{\sigma} C^{a}+d^{a}{ }_{I}^{m} F^{I} \sigma_{m}\right)+d^{a}{ }_{I}^{m} d_{m J K} F^{I J} \sigma^{K} .
$$

Adding a CS 4-form term to $G^{a}$

$$
G^{a}=d C^{a}+d^{a}{ }_{I}^{m} F^{I} B_{m}-\alpha d^{a}{ }_{I}^{m} d_{m J K} A^{I} F^{J} A^{K} .
$$

we find

$$
\begin{aligned}
\delta_{\sigma} G^{a}= & d\left[\delta_{\sigma} C^{a}+d^{a}{ }_{I}{ }^{m} F^{I} \sigma_{m}-\alpha d^{a}{ }_{I}{ }^{m} d_{m J K}\left(\sigma^{I} F^{J} A^{K}-A^{I} F^{J} \sigma^{K}\right)\right] \\
& +d^{a}{ }_{I}{ }^{m} d_{m J K}\left[\alpha \sigma^{I} F^{J K}+(1-\alpha) F^{I J} \sigma^{K}\right] .
\end{aligned}
$$

The last term can be made to vanish by simply requiring

$$
\alpha d^{a}{ }_{I}^{m} d_{m J K}=(\alpha-1) d_{(J \mid}^{a} d_{m \mid K) I} .
$$

Symmetrizing both sides of this equation w.r.t. $I J K$ we conclude that

$$
d_{(I \mid}^{a} d_{m \mid J K)}=0
$$

and going back to the original (unsymmetrized) equation this implies that $\alpha=1 / 3$. We arrive to the field strength, gauge transformation and Bianchi identities

$$
\begin{aligned}
G^{a} & =d C^{a}+d^{a}{ }_{I}{ }^{m} F^{I} B_{m}-\frac{1}{3} d^{a}{ }_{I}{ }^{m} d_{m J K} A^{I} F^{J} A^{K}, \\
\delta_{\sigma} C^{a} & =d \sigma^{a}-d^{a}{ }_{I}{ }^{m} F^{I} \sigma_{m}+\frac{1}{3} d^{a}{ }_{I}{ }^{m} d_{m J K}\left(\sigma^{I} F^{J} A^{K}-A^{I} F^{J} \sigma^{K}\right), \\
d G^{a} & =d^{a}{ }_{I}{ }^{m} F^{I} H_{m} .
\end{aligned}
$$

If these deformations are going to preserve formal symplectic invariance, we expect that these results extend to the dual 3 -forms and 4 -forms field strengths, that is:

$$
\begin{aligned}
G^{i} & =d C^{i}+d^{i}{ }_{I}{ }^{m} F^{I} B_{m}-\frac{1}{3} d^{i}{ }_{I}{ }^{m} d_{m J K} A^{I} F^{J} A^{K}, \\
\delta_{\sigma} C^{i} & =d \sigma^{i}-d^{i}{ }_{I}^{m} F^{I} \sigma_{m}+\frac{1}{3} d^{i}{ }_{I}{ }^{m} d_{m J K}\left(\sigma^{I} F^{J} A^{K}-A^{I} F^{J} \sigma^{K}\right), \\
d G^{i} & =d^{i}{ }_{I}{ }^{m} F^{I} H_{m}
\end{aligned}
$$

\footnotetext{
${ }^{17}$ The antisymmetric part is a total derivative that can be absorbed into a redefinition of $B_{m}$.
} 
while the identity

$$
d_{(I \mid}^{i} d_{m \mid J K)}=0
$$

This requires the introduction of new $\mathrm{CS}$ terms in the action. If we define the CS terms in the 4 -form field strengths by $\Delta G^{i}\left(G^{i}=d C^{i}+\Delta G^{i}\right)$, then we expect the following terms to be present:

$$
S_{C S}=-\int\left\{d C^{a} \Delta G_{a}+\frac{1}{2} \Delta G^{a} \Delta G_{a}\right\}
$$

Instead of checking in detail the gauge-invariance of these terms, it is more convenient to take the formal exterior derivative and check whether it is entirely given in terms of the gauge-invariant field strengths found above. If it is not, it should fail only by a total derivative which we can compensate by adding the corresponding terms to the action.

We have found that one has to relate $d_{(I \mid}^{i} d_{i \mid J)}{ }^{n}$ to the tensor $d_{m I J}$. The relation can be established by introducing a new tensor $d^{m n p}=-d^{n m p}$ and is given by

$$
d^{i}{ }_{(I \mid}^{m} d_{i \mid J)}^{n}=-2 d^{m n p} d_{p I J} .
$$

Observe that $d^{i}{ }_{[I \mid}^{m} d_{i \mid J]}{ }^{n}$ does not necessarily vanish.

Using the above relation we find a result of the expected form ${ }^{18}$

$$
\begin{aligned}
d\left\{d C^{a} \Delta G_{a}+\frac{1}{2} \Delta G^{a} \Delta G_{a}\right\}= & d_{a I}{ }^{m} G^{a} F^{I} H_{m}-\frac{1}{3} d^{m n p} H_{m n p}+d\left\{-\frac{1}{6} d^{m n p} B_{m} d B_{n} d B_{p}\right. \\
& \left.+\frac{1}{2} d^{m n p} B_{m} H_{n p}+\frac{1}{24} d_{I}^{i}{ }_{I}^{m} d_{i J}^{n} A^{I J} \Delta H_{m} d B_{n}\right\}
\end{aligned}
$$

from which it follows that the gauge-invariant CS term in the action is given, up to total derivatives, by

$$
\begin{aligned}
S_{C S}=\int\{ & -d C^{a} \Delta G_{a}-\frac{1}{2} \Delta G^{a} \Delta G_{a}-\frac{1}{6} d^{m n p} B_{m} d B_{n} d B_{p}+\frac{1}{2} d^{m n p} B_{m} H_{n p} \\
& \left.+\frac{1}{24} d^{i}{ }_{I}^{m} d_{i J}{ }^{n} A^{I J} \Delta H_{m} d B_{n}\right\} .
\end{aligned}
$$

Observe that only the completely antisymmetric part of $d^{m n p}$ enters the action, even though we have only assumed it to be antisymmetric in the first two indices. We will henceforth assume that $d^{m n p}$ is completely antisymmetric.

Now, as a final check of the consistency of our results, we can compute the dual field strengths $\tilde{H}^{m}$ and $\tilde{F}_{I}$, which should be formally symplectic invariant if the theory is, and their Bianchi identities, which should be given entirely in terms of other field strengths if the theory is indeed gauge invariant.

\footnotetext{
${ }^{18}$ We use repeatedly the identity

$$
2 d^{i}{ }_{I}^{m} d_{i J}{ }^{n} F^{I} A^{J} \Delta H_{n}=-6 d^{m n p} \Delta H_{n} \Delta H_{p}+d\left\{\frac{1}{2} d^{i}{ }_{I}^{m} d_{i J}{ }^{n} A^{I J} \Delta H_{n}\right\} .
$$
}


We find

$$
\begin{aligned}
\tilde{H}^{m}= & d \tilde{B}^{m}+d^{i}{ }_{I}^{m} C_{i} F^{I}+d^{m n p} B_{n}\left(H_{p}+\Delta H_{p}\right)+\frac{1}{12} d_{I}^{i}{ }_{I}^{m} d_{i J}{ }^{n} A^{I J} \Delta H_{n}, \\
d \tilde{H}^{m}= & d^{i}{ }_{I}^{m} G_{i} F^{I}+d^{m n p} H_{n p}, \\
\tilde{F}_{I}= & d \tilde{A}_{I}+2 d_{m I J} A^{J}\left(\tilde{H}_{m}-\frac{1}{2} \Delta \tilde{H}_{m}\right)-\left(d^{i}{ }_{I}^{m} B_{m}-\frac{1}{3} d^{i}{ }_{J}^{m} d_{m I K} A^{J K}\right)\left(G_{i}-\frac{1}{2} \Delta G_{i}\right) \\
& -\frac{1}{3}\left(d^{i}{ }_{I}^{m} d_{m J K}-d^{i}{ }_{K}^{m} d_{m I J}\right) F^{J} A^{K} C_{i}-d^{m n p} d_{m I J} A^{J} B_{n} H_{p} \\
& +\frac{1}{24}\left(d^{i} K^{m} d_{i L}{ }^{n} d_{m I J}+2 d^{i}{ }_{[I \mid}{ }^{m} d_{i \mid K]^{n}} d_{m J L}\right) F^{J} A^{K L} B_{n}+\frac{1}{24} d^{i}{ }_{J}^{m} d_{i K}^{n} d_{m I L} A^{J K L} d B_{n} \\
& -\frac{1}{180} d^{i} L^{n} d_{i Q}{ }^{m} d_{m I J} d_{n P K} A^{J K L Q} F^{P}, \\
d \tilde{F}_{I}= & 2 d_{m I J} F^{J} \tilde{H}^{m}+d_{i I}{ }^{m} G^{i} H_{m} .
\end{aligned}
$$

The duality relations are the same as in the undeformed case.

As a further check of this construction, taking the exterior derivative of the Bianchi identities of all the field strengths one finds consistent results upon use of the properties of the deformation tensors $d^{i} I^{m}, d_{m I J}, d^{m n p}$.

We will not compute the gauge transformations of the higher-rank form fields since they will not be necessary in what follows.

\subsection{The 6-form potentials and their 7-form field strengths}

On general grounds (see [28] and references therein) the 6-form potentials are expected to be the duals of the scalars. However, maintaining the manifest invariances of the theory in the dualization procedure requires the introduction of as many 6 -forms $D_{A}$ as generators of global transformations $\delta_{A}$ leaving the equations of motion (not just the action) invariant. Hence, the index $A$ labels the adjoint representation of the duality group. The 7-form field strengths $K_{A}$ are the Hodge duals of the piece $j_{A}^{(\sigma)}(\phi)$ of the Noether-Gaillard-Zumino (NGZ) conserved 1-form currents $j_{A}=j_{A}^{(\sigma)}(\phi)+\Delta j_{A}$ associated to those symmetries (or, better, dualities) [29] which only depend on the scalar fields ${ }^{19}$

$$
K_{A} \equiv-\star j_{A}^{(\sigma)}
$$

and their Bianchi identities follow from the conservation law for those currents

$$
d K_{A}=d \star j_{A}^{(\sigma)}=d \star\left(j_{A}^{N G Z}-\Delta j_{A}\right)=-d \star \Delta j_{A},
$$

where we have used the conservation of the NGZ current.

\footnotetext{
${ }^{19}$ This is the contribution of the $\sigma$-model to the Noether current. The symmetries of the equations of motion are necessarily symmetries of the $\sigma$-model, i.e. isometries of the $\sigma$-model metric $\mathcal{G}_{x y}(\phi)$ generated by Killing vectors $k_{A}{ }^{x}$. The indices $A, B, C$ label the symmetries of the theory and, therefore, run over the adjoint representation of the Lie algebra of that symmetry group G. The contribution of the $\sigma$-model to the NGZ 1-form is $j_{A}^{(\sigma)}=k_{A}{ }^{x} \mathcal{G}_{x y} d \phi^{y}$.
} 
The simplest procedure to compute $\Delta j_{A}$ is to contract the equations of motion of the scalars with the Killing vectors $k_{A}^{x}(\phi)$ of the $\sigma$-model metric $\mathcal{G}_{x y}(\phi)$, which is given by

$$
\begin{aligned}
\frac{\delta S}{\delta \phi^{x}}= & -d\left(\star \mathcal{G}_{x y} d \phi^{y}\right)+\frac{1}{2} \partial_{x} \mathcal{G}_{y z} d \phi^{y} \wedge \star d \phi^{z} \\
& +\frac{1}{2} \partial_{x} \mathcal{M}_{I J} F^{I} \wedge \star F^{J}+\frac{1}{2} \partial_{x} \mathcal{M}^{m n} H_{m} \wedge \star H_{n}+G^{a} \partial_{x} G_{a} .
\end{aligned}
$$

Using the Killing equation, we get

$$
k_{A}{ }^{x} \frac{\delta S}{\delta \phi^{x}}=-d \star j_{A}^{(\sigma)}+\frac{1}{2} k_{A}{ }^{x} \partial_{x} \mathcal{M}_{I J} F^{I} \wedge \star F^{J}+\frac{1}{2} k_{A}{ }^{x} \partial_{x} \mathcal{M}^{m n} H_{m} \wedge \star H_{n}+G^{a} k_{A}{ }^{x} \partial_{x} G_{a} .
$$

We must now use the fact that the isometry generated by $k_{A}$ will only be a symmetry of the equations of motion if ${ }^{20}$

$$
\begin{aligned}
k_{A}{ }^{x} \partial_{x} \mathcal{M}_{I J} & =-2 T_{A}{ }^{K}{ }_{(I} \mathcal{M}_{J) K}, \\
k_{A}{ }^{x} \partial_{x} \mathcal{M}^{m n} & =2 T_{A}{ }^{(m}{ }_{p} \mathcal{M}^{n) p} \\
k_{A}{ }^{x} \partial_{x} \mathcal{N}_{a b} & =-T_{A a b}-\mathcal{N}_{a c} T_{A}{ }^{c}{ }_{b}+T_{A a}{ }^{c} \mathcal{N}_{c b}+\mathcal{N}_{a c} T_{A}{ }^{c d} \mathcal{N}_{d b},
\end{aligned}
$$

where the matrices $T_{A}^{I}{ }_{J}, T_{A}{ }_{n}$ and

$$
\left(T_{A}{ }^{i}{ }_{j}\right) \equiv\left(\begin{array}{ll}
T_{A}{ }^{a}{ }_{b} & T_{A}{ }^{a b} \\
T_{A a b} & T_{A a}{ }^{b}
\end{array}\right),
$$

are generators of the symmetry group $\mathrm{G}$ in the representation in which the 1-forms, 2-forms and 3-forms transform

$$
\left[T_{A}, T_{B}\right]=f_{A B}{ }^{C} T_{C}, \quad\left[k_{A}, k_{B}\right]=-f_{A B}{ }^{C} k_{C} .
$$

As we have discussed, this implies that the matrices $T_{A}{ }_{j}{ }_{j}$ are generators of the symplectic group

$$
T_{A}^{i}{ }_{[j} \Omega_{k] i}=0 .
$$

Upon use of the duality relations between field strengths, we find that

$$
-k_{A}{ }^{x} \frac{\delta S}{\delta \phi^{x}}=d \star j_{A}^{(\sigma)}+T_{A}{ }_{I} F^{I} \tilde{F}_{J}+T_{A}{ }_{n}{ }_{n} \tilde{H}^{n} H_{m}-\frac{1}{2} T_{A i j} G^{i j}=0
$$

on shell. The exterior derivative of the whole expression vanishes due to the Bianchi identities of the field strengths and to the invariance of the deformation tensors $d_{m I J}, d_{I}^{i}{ }^{m}$ and $d^{m n p}$ under the $\delta_{A}$ transformations:

$$
\begin{aligned}
& \delta_{A} d_{m I J}=-T_{A}{ }^{n}{ }_{m} d_{n I J}-2 T_{A}{ }^{K}{ }_{(I \mid} d_{n \mid J) K}=0, \\
& \delta_{A} d^{i}{ }_{I}{ }^{m}=T_{A}{ }^{i}{ }_{j} d^{j}{ }_{I}{ }^{m}-T_{A}{ }^{J}{ }_{I} d^{i}{ }_{J}{ }^{m}+T_{A}{ }^{m}{ }_{n} d^{i}{ }_{I}{ }^{n}=0, \\
& \delta_{A} d^{m n p}=3 T_{A}{ }_{A}{ }^{m}{ }^{m \mid} d^{q \mid n p]}=0 .
\end{aligned}
$$

\footnotetext{
${ }^{20}$ The transformation rule for the period matrix is unconventional because our definition of the lower component of the symplectic vector of 4 -form field strengths, $G_{a}=R_{a}$ is unconventional (the sign is the oposite to the conventional one).
} 
This means that we can rewrite that equation locally as the conservation of the NGZ current

$$
d \star j_{A}^{N G Z}=0, \quad j_{A}^{N G Z} \equiv j_{A}^{(\sigma)}+\Delta j_{A},
$$

where $\Delta j_{A}$ is a very long and complicated expression whose explicit form will not be useful for us. A local solution is provided by $\star\left[j_{A}^{(\sigma)}+\Delta j_{A}\right]=-d D_{A}$ for the 6 -form potential $D_{A}$ and we get the definition of the 7-form field strength

$$
\star j_{A}^{(\sigma)}=-d D_{A}+\star \Delta j_{A} \equiv K_{A} .
$$

Its Bianchi identity is given by

$$
d K_{A}=-d \star j_{A}^{(\sigma)}=T_{A}^{I}{ }_{J} F^{J} \tilde{F}_{I}+T_{A}{ }_{n}{ }_{n} \tilde{H}^{n} H_{m}+\frac{1}{2} T_{A i j} G^{i j} .
$$

In the kind of theories that we are considering here there is no reason to include potentials of rank higher than 6 , unless we introduce a scalar potential depending on new coupling constants: one can then introduce 7 -form potentials dual to those coupling constants. Since the introduction of these parameters would be purely ad hoc, we will postpone the study of this duality to the next section in which we will be able to use in the definition of the scalar potential the embedding tensor and the massive deformation parameters, which have well-defined properties.

One can also generalize the theory by adding a scalar potential. This addition is associated to the introduction of new deformation parameters. In gauged supergravity, which is the main case of interest, these deformation parameters are the components of the embedding tensor and the scalar potential arises in the gauging procedure, associated to the fermion shifts in the fermion's supersymmetry transformations. Thus, it is natural to deal with the scalar potential in the next section too.

The results obtained in this and the previous section are summarized in appendix A.

\section{Non-Abelian and massive deformations: the tensor hierarchy}

The next step in the construction of the most general $d=8$ field theory is the gauging of the global symmetries of the theory. The most general possibilities can be explored using the embedding tensor formalism ${ }^{21}$ and in this section we are going to set it up for the Abelian theories we have just found. ${ }^{22}$ For the sake of convenience we are going to reproduce some of the formulae obtained above.

The starting point is the assumption that the equations of motion of the theory are invariant under a global symmetry group with infinitesimal generators $\left\{T_{A}\right\}$ satisfying the algebra

$$
\left[T_{A}, T_{B}\right]=f_{A B}{ }^{C} T_{C} .
$$

\footnotetext{
${ }^{21}$ In this section we will follow ref. [7], where the essential references on the embedding tensor formalism can be found. We will also use the same notation.

${ }^{22}$ Observer that, in general, the theories that we are considering are just the bosonic sector of a theory that also contains fermions and whose symmetry group may include symmetries that only act on them. The total symmetry group would, then, be larger and the embedding tensor should take this fact into account.
} 
The group acts linearly on all the forms of rank $\geq 1$, including the 3 -forms if the electric and magnetic 3-forms $C^{a}$ and $C_{a}$ are combined into a single symplectic vector of 3 -forms $\left(C^{i}\right)=\left(\begin{array}{l}C^{a} \\ C_{a}\end{array}\right)$ as explained above and codify electric-magnetic transformations involving the scalars. The matrices that represent the generators are denoted by $\left\{T_{A}{ }^{I} J\right\},\left\{T_{A}{ }_{n}{ }_{n}\right\},\left\{T_{A}{ }^{i} j\right\}$ and the adjoint generators are $T_{A}{ }^{B}{ }_{C}=f_{A C}{ }^{B}$. The matrices $T_{A}{ }^{i}{ }_{j}$ are generators of the symplectic group

$$
T_{A}^{i}{ }_{[j} \Omega_{k] i}=0, \quad\left(\Omega_{i j}\right)=\left(\Omega^{i j}\right) \equiv\left(\begin{array}{cc}
0 & \mathbb{1} \\
-\mathbb{1} & 0
\end{array}\right)
$$

We have

$$
\begin{array}{ll}
\delta_{\alpha} A^{I}=\alpha^{A} T_{A}{ }^{I}{ }_{J} A^{J}, & \delta_{\alpha} B_{m}=-\alpha^{A} T_{A}{ }^{n}{ }_{m} B_{n}, \quad \delta_{\alpha} C^{i}=\alpha^{A} T_{A}{ }_{j}{ }_{j} C^{j}, \\
\delta_{\alpha} \tilde{A}_{I}=-\alpha^{A} T_{A}{ }^{J}{ }_{I} \tilde{A}_{J}, & \delta_{\alpha} \tilde{B}^{m}=\alpha^{A} T_{A}{ }^{m}{ }_{n} \tilde{B}^{n},
\end{array}
$$

(the dual potentials transform in the dual covariant-contravariant representation).

The kinetic matrices $\mathcal{M}_{I J}, \mathcal{M}^{m n}, \mathcal{W}_{i j}(\mathcal{N})$ also transform linearly: if $\delta_{\alpha} \equiv \alpha^{A} \delta_{A}$

$$
\delta_{A} \mathcal{M}_{I J}=-2 T_{A}{ }_{(I} \mathcal{M}_{J) K}, \quad \delta_{A} \mathcal{M}^{m n}=2 T_{A}{ }^{(m}{ }_{p} \mathcal{M}^{n) p}, \quad \delta_{A} \mathcal{W}_{i j}=-2 T_{A}{ }_{(i}^{k} \mathcal{W}_{j) k},
$$

but the period matrix undergoes fractional-linear transformations which, infinitesimally, take the form

$$
\delta_{A} \mathcal{N}_{a b}=-T_{A a b}-\mathcal{N}_{a c} T_{A}{ }^{c}{ }_{b}+T_{A a}{ }^{c} \mathcal{N}_{c b}+\mathcal{N}_{a c} T_{A}{ }^{c d} \mathcal{N}_{d b}, \quad\left(T_{A}{ }^{i}{ }_{j}\right)=\left(\begin{array}{c}
T_{A}{ }^{a}{ }_{b} T_{A}{ }^{a b} \\
T_{A a b} T_{A a}{ }^{b}
\end{array}\right) .
$$

The $k$-form field strengths will transform in the same representation as the corresponding $(k-1)$-form potential, but only if the $d$-tensors $d_{m I J}, d^{i}{ }_{I}^{m}$ and $d^{m n p}$ are invariant under the global symmetry group, i.e. if they must satisfy

$$
\begin{aligned}
\delta_{A} d_{m I J} & =-T_{A}{ }^{n}{ }_{m} d_{n I J}-2 T_{A}{ }^{K}{ }_{(I \mid} d_{n \mid J) K}=0, \\
\delta_{A} d^{i}{ }_{I}{ }^{m} & =T_{A}{ }^{i}{ }_{j} d^{j}{ }_{I}{ }^{m}-T_{A}{ }^{J}{ }_{I} d^{i}{ }^{m}{ }^{m}+T_{A}{ }^{m}{ }_{n} d^{i}{ }_{I}{ }^{n}=0, \\
\delta_{A} d^{m n p} & =3 T_{A}{ }_{A}{ }_{q}{ }^{m \mid} d^{q \mid n p]}=0 .
\end{aligned}
$$

The theories we have constructed are invariant under Abelian gauge transformations with 0 -, 1- and 2-form parameters $\sigma^{I}, \sigma_{m}, \sigma^{i}$ :

$$
\delta_{\sigma} A^{I} \sim d \sigma^{I}, \quad \delta_{\sigma} B_{m} \sim d \sigma_{m}, \quad \delta_{\sigma} C^{i} \sim d \sigma^{i} .
$$

In order to gauge the global symmetries, we promote the global parameters $\alpha^{A}$ to local ones $\alpha^{A}(x)$ and we identify them with some combinations of the gauge parameters of the 1-forms $\sigma^{I}$ via the embedding tensor $\vartheta_{I}{ }^{A}$ as follows:

$$
\alpha^{A} \equiv \sigma^{I} \vartheta_{I}^{A} .
$$

Using this redefinition in the transformation of the kinetic matrices $\mathcal{M}_{I J}, \mathcal{M}^{m n}, \mathcal{W}_{i j}$ one immediately finds their gauge transformations:

$$
\delta_{\sigma} \mathcal{M}_{I J}=-2 \sigma^{L} X_{L}{ }^{K}{ }_{(I} \mathcal{M}_{J) K}, \quad \delta_{\sigma} \mathcal{M}^{m n}=2 \sigma^{I} T_{I}{ }^{(m}{ }_{p} \mathcal{M}^{n) p}, \quad \delta_{\sigma} \mathcal{W}_{i j}=-2 \sigma^{I} X_{I}{ }^{k}{ }_{i} \mathcal{W}_{j) k},
$$


where we have defined the matrices

$$
X_{I}{ }^{J}{ }_{K} \equiv \vartheta_{I}{ }^{A} T_{A}{ }^{J}{ }_{K}, \quad X_{I}{ }^{m}{ }_{n} \equiv \vartheta_{I}{ }^{A} T_{A}{ }_{n}{ }_{n} . \quad X_{I}{ }^{i}{ }_{j} \equiv \vartheta_{I}{ }^{A} T_{A}{ }^{i}{ }_{j} .
$$

The gauge fields for these symmetries are given by

$$
A^{A} \equiv A^{I} \vartheta_{I}^{A} .
$$

With them we can construct gauge-covariant derivatives, which we will then use to derive Bianchi identities.

Is is convenient to start by constructing the covariant derivatives of the kinetic matrices $\mathcal{M}_{I J}, \mathcal{M}^{m n}, \mathcal{W}_{i j}(\mathcal{N})$ which transform linearly. According to the general rule, the covariant derivative of a field $\Phi$ transforming as $\delta_{A} \Phi$ is given by

$$
\mathcal{D} \Phi \equiv d \Phi-A^{A} \delta_{A} \Phi .
$$

Then, with the above definition of gauge fields

$$
\begin{aligned}
\mathcal{D} \mathcal{M}^{m n} & =d \mathcal{M}^{m n}-2 A^{I} X_{I}{ }^{(m}{ }_{p} \mathcal{M}^{n) p}, \\
\mathcal{D} \mathcal{M}_{I J} & =d \mathcal{M}_{I J}+2 A^{L} X_{L}{ }^{K}{ }_{(I} \mathcal{M}_{J) K}, \\
\mathcal{D} \mathcal{W}_{i j} & =d \mathcal{W}_{i j}+2 A^{I} X_{I}{ }^{k}{ }_{(i} \mathcal{W}_{j) k} .
\end{aligned}
$$

These derivatives transform covariantly under gauge transformations $\delta_{\sigma}=\sigma^{I} \vartheta_{I}^{A} \delta_{A}$ provided that the embedding tensor is gauge-invariant

$$
\delta_{\sigma} \vartheta_{I}^{A}=0,
$$

and provided that the 1 -forms transform as

$$
\delta_{\sigma} A^{I}=\mathcal{D} \sigma^{I}+\Delta_{\sigma} A^{I}, \quad \text { where }\left\{\begin{aligned}
\Delta_{\sigma} A^{I} \vartheta_{I}{ }^{A} & =0, \\
\mathcal{D} \sigma^{I} & =d \sigma^{I}-A^{J} X_{J}{ }_{K}{ }^{K} \sigma^{K},
\end{aligned}\right.
$$

The condition eq. (3.16) leads to the so-called quadratic constraint

$$
\vartheta_{J}^{B}\left[T_{B}{ }^{K}{ }_{I} \vartheta_{K}^{A}-f_{B C}{ }^{A} \vartheta_{I}^{C}\right]=0 .
$$

To determine $\Delta_{\sigma} A^{I}$ we have to construct the gauge-covariant 2-form field strengths $F^{I}$.

\subsection{2-form field strengths}

The simplest way to find the 2-form field strengths $F^{I}$ is through the Ricci identities. A straightforward calculation using the quadratic constraint eq. (3.18) leads to

$$
\mathcal{D} \mathcal{D} \mathcal{M}_{m n}=-F^{I} \vartheta_{I}^{A} \delta_{A} \mathcal{M}_{m n},
$$

and analogous equations for $\mathcal{M}_{I J}$ and $\mathcal{W}_{i j}(\mathcal{N})$, with

$$
F^{I}=d A^{I}-\frac{1}{2} X_{J}^{I}{ }_{K} A^{J K}+\Delta F^{I}, \quad \text { where } \quad \Delta F^{I} \vartheta_{I}^{A}=0 .
$$


Under gauge transformations,

$$
\delta_{\sigma} F^{I}=\sigma^{J} X_{J}^{I}{ }_{K} F^{K},
$$

provided that

$$
\delta_{\sigma} \Delta F^{I}=-\mathcal{D} \Delta_{\sigma} A^{I}+2 X_{\left(J^{I} K\right)}\left(F^{J} \sigma^{K}-\frac{1}{2} A^{J} \delta_{\sigma} A^{K}\right) .
$$

Given the field content of the theory, the natural candidate to $\Delta F^{I}$ and $\Delta_{\sigma} A^{I}$ are

$$
\Delta F^{I}=Z^{I m} B_{m}, \quad \Delta_{\sigma} A^{I}=-Z^{I m} \sigma_{m},
$$

where the new tensor $Z^{I m}$ is gauge-invariant and orthogonal to the embedding tensor:

$$
\begin{aligned}
\delta_{\sigma} Z^{I m} & =0, \\
Z^{I m} \vartheta_{I}^{A} & =0 .
\end{aligned}
$$

Then, the consistency of eq. (3.22) with the above choice requires

$$
\vartheta_{(J \mid}^{A} T_{A}^{I}{ }_{\mid K)}=Z^{I m} d_{m J K},
$$

for some tensor $d_{m J K}=d_{m K J}$ which will turn out to coincide with the tensor we introduced as an Abelian deformation in the previous sections.

We conclude that ${ }^{23}$

$$
\begin{aligned}
F^{I} & =d A^{I}-\frac{1}{2} X_{J}^{I}{ }_{K} A^{J K}+Z^{I m} B_{m}, \\
\delta_{\sigma} F^{I} & =\sigma^{J} X_{J}^{I}{ }_{K} F^{K}, \\
\delta_{\sigma} A^{I} & =\mathcal{D} \sigma^{I}-Z^{I m} \sigma_{m}, \\
\delta_{\sigma} B_{m} & =\mathcal{D} \sigma_{m}+2 d_{m J K}\left(F^{J} \sigma^{K}-\frac{1}{2} A^{J} \delta_{\sigma} A^{K}\right)+\Delta_{\sigma} B_{m}, \text { with } Z^{I m} \Delta_{\sigma} B_{m}=0 .
\end{aligned}
$$

In the ungauged limit $\vartheta_{I}{ }^{A}=Z^{I m}=0$ we get the Abelian gauge transformations of the 2-form eq. (2.21) if we identify the above 1 -form $\sigma_{m}$ (relabeled $\sigma_{\mathrm{g} m}$ ) with $\sigma_{m}-d_{m I J} A^{I} \sigma^{J}$,

$$
\sigma_{\mathrm{g} m}=\sigma_{m}-d_{m I J} A^{I} \sigma^{J},
$$

confirming the identification of the $d$-tensor. Using that variable makes the non-Abelian gauge transformations more complicated and, therefore, we will stick to the above $\sigma_{m}$.

\subsection{3 -form field strengths}

Again, the shortest way to find $\Delta B_{m}$ and the gauge-covariant 3-form field strength $H_{m}$ is through the Bianchi identities. Taking the covariant derivative of the 2 -form field strength, and using the generalized Jacobi identity

$$
X_{[I \mid}{ }^{K}{ }_{M} X_{\mid J}^{M}{ }_{L]}=\frac{2}{3} Z^{K m} d_{m M[I} X_{J}^{M}{ }_{L]}
$$

\footnotetext{
${ }^{23}$ On general grounds, we expect a term of the form $-\sigma^{I} X_{I}{ }^{n}{ }_{m} B_{n}$ in the gauge transformation rule of $B_{m}$. This term is indeed present, but in a disguised form.
} 
we get

$$
\mathcal{D} F^{I}=Z^{I m} H_{m},
$$

where

$$
H_{m}=\mathcal{D} B_{m}-d_{m I J} d A^{I} A^{J}+\frac{1}{3} d_{m M I} X_{J}{ }_{K}{ }_{K} A^{I J K}+\Delta H_{m}, \quad \text { with } \quad Z^{I m} \Delta H_{m}=0 .
$$

In the ungauged limit $\vartheta_{I}^{A}=Z^{I m}=0$ we recover the Abelian 3-form field strength in eq. (2.20). On the other hand, by construction, the above field strength is gauge-covariant up to terms annihilated by $Z^{I m}$ under eqs. (3.29) and (3.30). To show this explicitly, we will need further identities between the tensors of the theory that are more easily discovered by computing first the 4 -form field strengths.

\subsection{4 -form field strengths}

From this moment, following ref. [13], we will determine the general form of the field strengths using the Bianchi identities and their consistency relations. This procedure yields gauge-covariant field strengths and one can later find explicitly the gauge transformations of the fields that produce that result.

Thus, we take the covariant derivative of both sides of eq. (3.33), use the Ricci identity eq. (3.19) for the l.h.s. and the explicit form of $H_{m}$ in eq. (3.34) for the r.h.s., and we find the Bianchi identity for $H_{m}$ to be

$$
\mathcal{D} H_{m}=-d_{m I J} F^{I J}+\Delta \mathcal{D} H_{m}, \quad \text { where } Z^{I m} \Delta \mathcal{D} H_{m}=0 .
$$

$\Delta \mathcal{D} H_{m}$ has to be gauge-invariant and scalar-independent and the only possibility is a 4 form combination of field strengths. $F^{I} \wedge F^{J}$ has already been used and we must use $G^{i}$, whose explicit form will be determined by consistency. We need to introduce a new gauge-invariant tensor $Z_{i m}$ orthogonal to $Z^{I m}$

$$
Z^{I m} Z_{j m}=0,
$$

and, then, we arrive to the Bianchi identity

$$
\mathcal{D} H_{m}=-d_{m I J} F^{I J}+Z_{i m} G^{i} .
$$

A direct calculation of $\mathcal{D} H_{m}$ using the explicit expression of $H_{m}$ in eq. (3.34) with $\Delta H_{m}=Z_{i m} C^{i}$ can only give a consistent result if we introduce a tensor $d^{i} I^{m}$ such that

$$
X_{I}^{m}{ }_{n}+2 d_{n I J} Z^{J m}=Z_{i n} d_{I}^{i}{ }^{m} .
$$

The tensor $d^{i}{ }^{m}$ coincides with the one we introduced as an Abelian deformation.

We get

$$
G^{i}=\mathcal{D} C^{i}+d^{i}{ }^{n}\left[F^{I} B_{n}-\frac{1}{2} Z^{I p} B_{n} B_{p}-\frac{1}{3} d_{n J K} d A^{J} A^{I K}+\frac{1}{12} d_{n M J} X_{K}{ }^{M}{ }_{L} A^{I J K L}\right]+\Delta G^{i},
$$


with

$$
Z_{i m} \Delta G^{i}=0 .
$$

These 4-form field strengths reduce exactly to the Abelian ones in eq. (2.31).

Now we are ready to check explicitly using the identity/constraint eq. (3.38) that $H_{m}$ in eq. (3.34) with $\Delta H_{m}=Z_{i m} C^{i}$ is gauge covariant up to terms proportional to $Z_{i m}$, which are automatically annihilated by $Z^{I m}$. We find that

$$
\delta_{\sigma} C^{i}=\mathcal{D} \sigma^{i}-d_{I}^{i}{ }^{n}\left[\sigma^{I} H_{n}+F^{I} \sigma_{n}+\delta_{\sigma} A^{I} B_{n}-\frac{1}{3} d_{n J K} \delta_{\sigma} A^{J} A^{I K}\right]+\Delta_{\sigma} C^{i},
$$

with $Z_{i m} \Delta_{\sigma} C^{i}=0$,

$$
\Delta_{\sigma} B_{m}=-Z_{i m} \sigma^{i} \text {. }
$$

These gauge transformations reduce to the Abelian ones eq. (2.32) upon use of the property of the $d$-tensors eq. (2.34) and the identifications eq. (3.31) and

$$
\sigma_{\mathrm{g}}^{i}=\sigma^{i}+d_{I}^{i}\left(B_{n} \sigma^{I}-\frac{1}{3} d_{n J K} A^{I J} \sigma^{K}\right) .
$$

\subsection{5 -form field strengths}

Taking, once again, the covariant derivatives of both sides of the Bianchi identity for $H_{m}$, eq. (3.37), and using the Bianchi identity for $F^{I}$, eq. (3.33) and the newly introduced tensor $d^{i} I^{m}$, we find that

$$
\mathcal{D} G^{i}=d^{i}{ }_{I}^{m} F^{I} H_{m}-Z^{i}{ }_{m} \tilde{H}^{m},
$$

where $Z_{m}^{i}$ is a new gauge-invariant tensor orthogonal to $Z_{i m}$

$$
Z_{m}^{i} Z_{i n}=0
$$

and where the sign of that term has been chosen so as to get the same signs as in the ungauged case. In principle these two tensors could be completely unrelated (except for the constraints). However, since, in the physical theory, $G^{i}$ is self-dual and $\tilde{H}^{m}$ is the electric-magnetic dual of $H_{m}$, it is natural to expect that the same tensors appear in both field strengths. Thus, we are going to assume that $Z_{m}^{i}$ has been obtained from $Z_{j m}$ by raising the index with the symplectic metric tensor $\Omega^{i j}$, that is

$$
Z^{i}{ }_{m} \equiv \Omega^{j i} Z_{j m} .
$$

Then, there is no new constraint associated to its gauge invariance and, we just have the constraint eq. (3.44) analogous to a constraint satisfied by the embedding tensor in 4-dimensional field theories.

\subsection{6-form field strengths}

Taking the covariant derivative of both sides of the Bianchi identity for $G^{i}$, eq. (3.43) and using the Bianchi identities for the field strengths of lower rank, we find that we need to 
introduce three new tensors $d_{i I}^{m}, d^{m n p}, d^{m}{ }_{I J K}$ and demand that

$$
\begin{aligned}
d_{I}^{i}{ }_{I}^{m} Z_{j m}+X_{I}{ }^{i}{ }_{j} & =-Z^{i}{ }_{m} d_{j I}{ }^{m}, \\
d^{i}{ }_{I}{ }^{[m \mid} Z^{I \mid n]} & =-Z^{i}{ }_{p} d^{p m n}, \\
d_{(I \mid}^{i}{ }^{m} d_{m \mid J K)} & =-Z^{i}{ }_{p} d^{p}{ }_{I J K} .
\end{aligned}
$$

Lowering the $i$ indices in the first equation with $\epsilon_{i k}$ and taking into account that $X_{I[k j]}=0$, we conclude that it is natural to identify

$$
d_{i I}^{m}=\Omega_{i j} d_{I}^{j},
$$

and rewrite the constraint as

$$
X_{I i j}=-2 Z_{(i \mid m} d_{\mid j) I}^{m} .
$$

Using these constraints and the same reasoning as in the previous cases we find the next Bianchi identity and we can also solve it ${ }^{24}$

$$
\begin{aligned}
\mathcal{D} \tilde{H}^{m}= & -d_{i I}{ }^{m} G^{i} F^{I}+d^{m n p} H_{n p}+d^{m}{ }_{I J K} F^{I J K}+Z^{I m} \tilde{F}_{I}, \\
\tilde{H}^{m}= & \mathcal{D} \tilde{B}^{m}-d_{i I}{ }^{m} F^{I} C^{i}+2 d^{m n p} B_{n}\left(H_{p}-Z_{i p} C^{i}-\frac{1}{2} \mathcal{D} B_{p}\right) \\
& +d^{m}{ }_{I J K} d A^{I} d A^{J} A^{K} \\
& +\left(\frac{1}{12} d_{i J}{ }^{m} d^{i} K^{n} d_{n I L}-\frac{3}{4} d^{m}{ }_{I J M} X_{K}{ }^{M}{ }_{L}\right) d A^{I} A^{J K L} \\
& +\left(\frac{3}{20} d^{m}{ }_{N P M} X_{I}{ }^{N}{ }_{J}-\frac{1}{60} d_{i M}{ }^{m} d_{I}^{i n} d_{n P J}\right) X_{K}{ }^{P}{ }_{L} A^{I J K L M} \\
& +Z^{I m} \tilde{A}_{I},
\end{aligned}
$$

\subsection{7-form field strengths}

Provided that we impose the additional constraint ${ }^{25}$

$$
d_{(I}^{i} d_{|i| J)}^{n}+2 d^{m n p} d_{p I J}+3 d^{m}{ }_{I J K} Z^{K n}=+3 d^{n}{ }_{I J K} Z^{K m},
$$

the covariant derivative of the Bianchi identity eq. (3.51) leads to the Bianchi identity for the 6 -form field strengths

$$
\begin{aligned}
\mathcal{D} \tilde{F}_{I}= & 2 d_{m I J} F^{J} \tilde{H}^{m}+d_{i I}{ }^{m} G^{i} H_{m}-3 d^{m}{ }_{I J K} F^{J K} H_{m}-\vartheta_{I}{ }^{A} K_{A} \\
\tilde{F}_{I}= & \mathcal{D} \tilde{A}_{I}+2 d_{m I J} \tilde{B}^{m} F^{J}+d_{i I}{ }^{m} C^{i}\left(H_{m}-\frac{1}{2} Z_{j m} C^{j}\right) \\
& -3 d^{m}{ }_{I J K} B_{m}\left(F^{J}-\frac{1}{2} Z^{J n} B_{n}\right)\left(F^{K}-\frac{1}{2} Z^{K p} B_{p}\right)-\frac{1}{4} d^{m}{ }_{I J K} Z^{J n} Z^{K p} B_{m n p} \\
& +\frac{1}{2} d_{i I}^{m} d^{i}{ }_{J}^{n}\left(F^{J}-\frac{2}{3} Z^{J p} B_{p}\right) B_{m n}-d_{i I}{ }^{m} B_{m} \square G^{i}+\cdots
\end{aligned}
$$

where we are denoting by $\square G^{i}$ the part of $G^{i}$ that only contains 1 -forms $A^{I}$ and their derivatives $d A^{I}$.

\footnotetext{
${ }^{24}$ Actually, it is easier to find $\tilde{H}^{m}$ from the previous Bianchi identity eq. (3.43) taking the covariant derivative of the 4 -form field strengths $G^{i}$ in eq. (3.39) with $\Delta G^{i}=-Z^{i}{ }_{m} \tilde{B}^{m}$.

${ }^{25}$ This constraint reduces to eq. (2.36) in the ungauged, massless limit.
} 


\subsection{8-form field strengths}

Taking the covariant derivative of eq. (3.54) and using several of the constraints imposed above, we find that

$$
\vartheta_{I}^{A} \mathcal{D} K_{A}=X_{I}{ }_{J} F^{J} \tilde{F}_{K}+X_{I}{ }_{n}^{m} \tilde{H}^{n} H_{m}+\frac{1}{2} X_{I i j} G^{i j}+5 d_{m(I J} d^{m}{ }_{K L M)} F^{J K L M} .
$$

According to the general arguments in ref. [28] the last term must vanish. It cannot arise in the Bianchi identity of the dual Noether-Gaillard-Zumino current associated to the global symmetries of the theory. Thus, we impose

$$
d_{m(I J} d_{K L M)}^{m}=0,
$$

and, from the definition of the $X$ tensors, we get

$$
\mathcal{D} K_{A}=T_{A}{ }_{J} F^{J} \tilde{F}_{K}+T_{A}{ }_{n}{ }_{n} \tilde{H}^{n} H_{m}-\frac{1}{2} T_{A i j} G^{i j}+Y_{A}^{\sharp} L_{\sharp},
$$

where $Y_{A}^{\sharp}$ is a tensor orthogonal to the embedding tensor

$$
\vartheta_{I}^{A} Y_{A}^{\sharp}=0,
$$

and where the index $\sharp$ runs over all the deformation tensors introduced so far, that we are going to denote collectively by $c^{\sharp}$. As argued in ref. [13], the natural candidates for the $Y_{A}^{\sharp}$ tensors are the variations of the deformation tensors $c^{\sharp}$ under the global symmetries of the theory

$$
Y_{A}^{\sharp}=\delta_{A} c^{\sharp},
$$

where $A$ runs over the whole Lie algebra of the global symmetry group, because all the deformation tensors are required to be gauge invariant

$$
\vartheta_{I}^{A} \delta_{A} c^{\sharp}=\vartheta_{I}^{A} Y_{A}^{\sharp} \equiv \mathcal{Q}_{I}^{\sharp}=0,
$$

where we have defined the constraints $\mathcal{Q}_{A}^{\sharp}$.

At this point there are two possibilities:

1. We can consider that all the independent tensors ${ }^{26}\left\{\vartheta_{I}{ }^{A}, Z^{I m}, Z_{i m},-d_{m I J}, d^{i} I^{m}\right\}$ are deformations of the original theory introduced at the same time as the gauging of the global symmetries of the original symmetry is carried out. In this case they only have to be invariant under the global symmetries that have been gauged and not the stronger condition

$$
\delta_{A} c^{\sharp}=0,
$$

for any of them.

\footnotetext{
${ }^{26}$ The tensors $d^{m n p}, d^{m}{ }_{I J K}$ are related to these and their gauge invariance is not an independent condition.
} 
2. We can consider only the tensors $\left\{\vartheta_{I}^{A}, Z^{I m}, Z_{i m}\right\}$ are deformations of the original theory, whose definition includes the tensors $\left\{-d_{m I J}, d^{i} I^{m}\right\}$. In this case, the latter must be invariant under the whole global symmetry group by hypothesis. The corresponding $Y_{A}^{\sharp}$ tensors are assumed to vanish identically, before they are contracted with the embedding tensor. This is the point of view that we have adopted here and it implies that there are only three sets of 8-form field strengths $\left\{L_{\sharp}\right\}=\left\{L_{A}^{I}, L_{I m}, L^{i m}\right\}$ and only three corresponding sets of 7 -form potentials $\left\{E_{\sharp}\right\}=\left\{E_{A}^{I}, E_{I m}, E^{i m}\right\}$ which are dual to the deformation tensors $\left\{\vartheta_{I}^{A}, Z^{I m}, Z_{i m}\right\}$. In an action in which these tensors are generalized to spacetime-dependent fields, these dual potentials appear as Lagrange multipliers enforcing their constancy [10, 30].

We, thus, have to consider three constraints associated to gauge invariance

$$
\begin{aligned}
\mathcal{Q}_{I J}{ }^{A} & \equiv \vartheta_{I}{ }^{B} Y_{B J}{ }^{A}, & Y_{B J}{ }^{A} & \equiv \delta_{B} \vartheta_{J}{ }^{A}=-T_{B}{ }^{K}{ }_{J} \vartheta_{K}{ }^{A}+T_{B}{ }^{A}{ }_{C} \vartheta_{J}{ }^{C}, \\
\mathcal{Q}_{I}{ }^{J m} & \equiv \vartheta_{I}{ }^{B} Y_{B}{ }^{J m}, & Y_{B}{ }^{J m} & \equiv \delta_{B} Z^{J m}=T_{B}{ }^{J}{ }_{K} Z^{K m}+T_{B}{ }^{m}{ }_{n} Z^{J n}, \\
\mathcal{Q}_{\text {Iim }} & \equiv \vartheta_{I}{ }^{B} Y_{\text {Bim }}, & Y_{\text {Bim }} & \equiv \delta_{B} Z_{i m}=-T_{B}{ }^{j}{ }_{i} Z_{j m}-T_{B}{ }^{n}{ }_{m} Z_{\text {in }},
\end{aligned}
$$

and two constraints associated to global invariance

$$
\begin{aligned}
& \mathcal{Q}_{A m I J} \equiv Y_{A m I J}=-\delta_{A} d_{m I J}=T_{A}{ }^{n}{ }_{m} d_{n I J}+2 T_{A}^{K}\left({ }_{(I} d_{|m| J) K},\right. \\
& \mathcal{Q}_{A}{ }^{i} I^{m}{ }^{m} \equiv Y_{A}{ }^{i} I^{m}=\delta_{A} d^{i}{ }_{I}{ }^{m}=T_{A}{ }^{i}{ }_{j} d^{j}{ }_{I}{ }^{m}-T_{A}{ }^{J}{ }_{I} d^{i}{ }_{J}{ }^{m}+T_{A}{ }^{m}{ }_{n} d^{i}{ }_{I}{ }^{n},
\end{aligned}
$$

and the final form of the Bianchi identity for the 7 -form field strengths is

$$
\mathcal{D} K_{A}=T_{A}{ }_{J} F^{J} \tilde{F}_{K}+T_{A}{ }_{n}{ }_{n} \tilde{H}^{n} H_{m}-\frac{1}{2} T_{A i j} G^{i j}+Y_{A I}{ }^{B} L_{B}{ }^{I}+Y_{A}{ }^{I m} L_{I m}+Y_{A i m} L^{i m} .
$$

The occurrence of these $Y_{A}^{\sharp}$ has to be confirmed by taking again the covariant derivative of this Bianchi identity.

\subsection{9 -form field strengths}

Taking the covariant derivative of both sides of the Bianchi identity eq. (3.68) we arrive to ${ }^{27}$

$$
\begin{aligned}
Y_{A I}{ }^{B}\left[\mathcal{D} L_{B}{ }^{I}+F^{I} K_{B}\right]+Y_{A}{ }^{I m}\left[\mathcal{D} L_{I m}\right. & \left.+\tilde{F}_{I} H_{m}\right]+Y_{A i m}\left[\mathcal{D} L^{i m}+G^{i} \tilde{H}^{m}\right] \\
& +\mathcal{Q}_{A m I J} \tilde{H}^{m} F^{I J}+\mathcal{Q}_{A}{ }^{i}{ }^{m} G_{i} F^{I} H_{m}=0 .
\end{aligned}
$$

Since we have assumed ${ }^{28} \mathcal{Q}_{A m I J}=\mathcal{Q}_{A}{ }^{i}{ }^{m}=0$, we arrive to the Bianchi identities

$$
\begin{aligned}
& \mathcal{D} L_{B}{ }^{I}=-F^{I} K_{B}-W_{B}^{I \beta} M_{\beta}, \\
& \mathcal{D} L_{I m}=-\tilde{F}_{I} H_{m}-W_{I m}^{\beta} M_{\beta}, \\
& \mathcal{D} L^{i m}=-G^{i} \tilde{H}^{m}-W^{i m \beta} M_{\beta},
\end{aligned}
$$

\footnotetext{
${ }^{27} \mathrm{By}$ direct computation we have not found any constraint or $Y_{A}^{\sharp}$ tensor associated to either $d^{m}{ }_{I J K}$ or $d^{m n p}$.

${ }^{28}$ Observe that, the alternative assumption is equally valid and can be made to work by including the 8-form field strengths $L^{m I J}, L_{i}{ }^{I} m$.
} 
where the $W_{\sharp}^{\beta}$ tensors are invariant tensors annihilated by the $Y_{A}^{\sharp}$ ones

$$
Y_{A}^{\sharp} W_{\sharp}^{\beta}=0 \text {. }
$$

As shown in ref. [13] these tensors are nothing but the derivatives of all the constraints satisfied by the deformation tensors (labeled by $\beta$ ) with respect to the deformation tensors themselves. This means that there are as many 9 -form field strengths $M_{\beta}$ and corresponding 8-form potentials $N_{\beta}$ as constraints $\mathcal{Q}^{\beta}=0$. In a general action the top-form potentials $N_{\beta}$ would occur as the Lagrange multipliers enforcing the constraints $\mathcal{Q}^{\beta}=0$.

As usual, this can be confirmed by acting yet again with the covariant derivative on the above three Bianchi identities. Let us first list all the constraints we have met:

1. First of all we have the gauge-invariance constraints

$$
\mathcal{Q}_{I J}{ }^{A}, \mathcal{Q}_{I}{ }^{J m}, \mathcal{Q}_{\text {Iim }}
$$

defined in eqs. (3.63)-(3.65).

2. Secondly, we have the global-invariance constraints

$$
\mathcal{Q}_{A m I J}, \mathcal{Q}_{A}^{i}{ }^{m},
$$

defined in eqs. (3.66) and (3.67).

3. Thirdly we have the orthogonality constraints between the three deformation tensors

$$
\begin{aligned}
\mathcal{Q}^{m A} & \equiv-Z^{I m} \vartheta_{I}{ }^{A}, \\
\mathcal{Q}_{i}{ }^{I} & \equiv Z_{i m} Z^{I m} \\
\mathcal{Q}_{m n} & \equiv Z_{i m} Z^{i}{ }_{n} .
\end{aligned}
$$

4. Next, we have the constraints relating the gauge transformations to the $d$-tensors

$$
\begin{aligned}
\mathcal{Q}_{I}{ }^{J}{ }_{K} & \equiv X_{(I}{ }^{J}{ }_{K)}-Z^{J m} d_{m I K}, \\
\mathcal{Q}_{I}{ }^{m}{ }_{n} & \equiv X_{I}{ }_{n}{ }_{n}+2 d_{n I J} Z^{J m}+Z_{\mathrm{in}} d^{i}{ }^{m}, \\
\mathcal{Q}_{I i j} & \equiv-X_{I i j}-2 Z_{(i \mid m} d_{\mid j) I}{ }^{m}
\end{aligned}
$$

5. Finally, we have the constraints that related the $d$-tensors amongst them via the massive deformations $Z$

$$
\begin{aligned}
\mathcal{Q}^{i m n} & \equiv d^{i}{ }_{I}{ }^{[m \mid} Z^{I \mid n]}+Z_{p}^{i} d^{p m n}, \\
\mathcal{Q}_{I J}{ }^{m n} & \equiv \frac{1}{2} d_{(I \mid}^{{ }^{m}} d_{i \mid J)}{ }^{n}+d^{m n p} d_{p I J}+3 d^{[m \mid}{ }_{I J K} Z^{K \mid n]}, \\
\mathcal{Q}_{i I J K} & \equiv Z_{i m} d^{m}{ }_{I J K}-d_{i(I \mid}{ }^{m} d_{m \mid J K)} .
\end{aligned}
$$


From eq. (3.70) we get

$$
\begin{array}{r}
\frac{\partial \mathcal{Q}_{I J}{ }^{A}}{\partial \vartheta_{K}{ }^{B}}\left[\mathcal{D} M_{A}^{I J}{ }_{A}+F^{I} L_{A}{ }^{J}\right]+\frac{\partial \mathcal{Q}^{m A}}{\partial \vartheta_{K}^{B}}\left[\mathcal{D} M_{m A}+H_{m} K_{A}\right] \\
+\frac{\partial \mathcal{Q}_{I}{ }^{J} K}{\partial \vartheta_{K}{ }^{B}}\left[\mathcal{D} M_{J}^{I}{ }^{K}+F^{I K} \tilde{F}_{J}\right]+\frac{\partial \mathcal{Q}_{I}^{m}{ }_{n}}{\partial \vartheta_{K}^{B}}\left[\mathcal{D} M_{m}^{I}{ }^{n}+F^{I} \tilde{H}^{m} H_{n}\right] \\
+\frac{\partial \mathcal{Q}_{I i j}}{\partial \vartheta_{K}{ }^{B}}\left[\mathcal{D} M^{I i j}+F^{I} G^{i j}\right]=0 .
\end{array}
$$

From eqs. (3.71) and (3.72) we get very similar equations which guarantee the consistency of the whole construction of the tensor hierarchy that we have carried out in this section.

\section{Gauge-invariant action for the 1-, 2- and 3-forms}

The Bianchi identities of the full tensor hierarchy give rise to the equations of motion of the electric fields of the theory upon use of the duality relations (on-duality-shell). For field strengths of the 6-,5-, 4-forms they are given by

$$
K_{A}=-\star j_{A}^{(\sigma)}, \quad \tilde{F}_{I}=\mathcal{M}_{I J} \star F^{J}, \quad \tilde{H}^{m}=\star \mathcal{M}^{m n} H_{n} .
$$

For the field strengths of the magnetic 3-forms they are given by

$$
G_{a}=R_{a}
$$

where $R_{a}$ has been defined in eq. (1.5). Finally, the field strength of the 7 -forms is, according to refs. $[10,13]$, dual to the derivatives of the gauge-invariant scalar potential with respect to the deformation parameters, denoted collectively by $c^{\sharp}$

$$
L_{\sharp}=\star \frac{\partial V}{\partial c^{\sharp}} .
$$

This identity follows from the scalar equation of motion in presence of a scalar potential together with the condition

$$
k_{A}{ }^{x} \frac{\partial V}{\partial \phi^{x}}=Y_{A}^{\sharp} \frac{\partial V}{\partial c^{\sharp}},
$$

which implies, after multiplication by the embedding tensor $\vartheta_{I}{ }^{A}$, the gauge-invariance of the scalar potential.

In general, the equations of motion are combinations of different Bianchi identities on-duality-shell. In order to determine the combinations that correspond to the equations of motion we have to examine which combinations of Bianchi identities satisfy the Noether identities associated to the gauge invariances of the theory.

To start with, we need to introduce some notation for the Bianchi identities. This has been done in appendix B.2. These Bianchi identities are related by a hierarchy of identities that are obtained by taking the covariant derivative of those with lower rank, as we have shown. These identities of Bianchi identities are collected in appendix B.3. 
Now, let us assume that a standard gauge-invariant action for the 0 -forms $\mathcal{M}$ (or $\phi^{x}$ ), 1 forms $A^{I}, 2$-forms $B_{m}$ and electric 3-forms $C^{a}$ exists. This means that the Bianchi identities $\mathcal{B}\left(\mathcal{Q}^{\beta}\right), \mathcal{B}\left(c^{\sharp}\right)$ and $\mathcal{B}(\mathcal{D} \mathcal{M}), \mathcal{B}\left(F^{I}\right), \mathcal{B}\left(H_{m}\right), \mathcal{B}\left(G_{a}\right)$ are satisfied, at least up to duality relations. The kinetic terms of the electric fields are written in terms of the gauge-invariant field strengths and this implies that the magnetic fields $C_{a}, \tilde{B}^{m}$ must necessarily occur in the action, albeit not as dynamical fields: their equations of motion will be trivial on-duality-shell.

Under these assumptions, the identities satisfied by the non-trivial Bianchi identities (i.e. those involving the magnetic field strengths) take the simplified form ${ }^{29}$

$$
\begin{aligned}
\mathcal{D B}\left(H_{m}\right)-Z^{a}{ }_{m} G_{a} & =0, \\
\mathcal{D B}\left(G_{a}\right)-Z_{a m} \mathcal{B}\left(\tilde{H}^{m}\right) & =0, \\
\mathcal{D} \mathcal{B}\left(\tilde{H}^{m}\right)+d^{a}{ }_{I}{ }^{m} \mathcal{B}\left(G_{a}\right) F^{I}+Z^{I m} \mathcal{B}\left(\tilde{F}_{I}\right) & =0, \\
\mathcal{D} \mathcal{B}\left(\tilde{F}_{I}\right)+2 d_{m I J} \mathcal{B}\left(\tilde{H}^{m}\right) F^{J}-d^{a}{ }_{I}{ }^{m} \mathcal{B}\left(G_{a}\right) H_{m}+\vartheta_{I}{ }^{A} \mathcal{B}\left(K_{A}\right) & =0 .
\end{aligned}
$$

If such an action exists, its invariance with respect to gauge transformations with parameters $\sigma^{m}, \sigma^{i}, \sigma_{m}, \sigma^{I}$ will imply that the equations of motion satisfy, off-shell, associated Noether identities. Up to the field equations of $\tilde{B}^{m}$ and $C_{a}$ which are assumed to be satisfied up to dualities, they take the form

$$
\begin{aligned}
& \mathcal{D} \frac{\delta S}{\delta \tilde{B}^{m}}-Z^{a}{ }_{m} \frac{\delta S}{\delta C_{a}}=0, \\
& \mathcal{D} \frac{\delta S}{\delta C^{a}}-Z_{a m} \frac{\delta S}{\delta B_{m}}=0, \\
& \mathcal{D} \frac{\delta S}{\delta B_{m}}+Z^{I m}\left[\frac{\delta S}{\delta A^{I}}-d_{n I J} A^{J} \frac{\delta S}{\delta B_{n}}-\left(d^{a}{ }_{I}^{n} B_{n}-\frac{1}{3} d^{a}{ }^{n}{ }^{n} d_{n I K} A^{J K}\right) \frac{\delta S}{\delta C^{a}}\right] \\
& +d^{a}{ }_{I}^{m} F^{I} \frac{\delta S}{\delta C^{a}}=0 \\
& \mathcal{D}\left[\frac{\delta S}{\delta A^{I}}-d_{n I J} A^{J} \frac{\delta S}{\delta B_{n}}-\left(d^{a}{ }_{I}^{n} B_{n}-\frac{1}{3} d^{a}{ }_{J}^{n} d_{n I K} A^{J K}\right) \frac{\delta S}{\delta C^{a}}\right] \\
& +2 d_{m I J} F^{J} \frac{\delta S}{\delta B_{m}}+d^{a}{ }_{I}^{m} H_{m} \frac{\delta S}{\delta C^{a}}+\vartheta_{I}{ }^{A} k_{A}{ }^{x} \frac{\delta S}{\delta \phi^{x}}=0 .
\end{aligned}
$$

Comparing directly with the above identities satisfied by the Bianchi identities, we conclude that, up to dualities, the equations of motion of the electric fields are related to the Bianchi identities of the magnetic field strengths by

$$
\begin{aligned}
k_{A}{ }^{x} \frac{\delta S}{\delta \phi^{x}} & =\mathcal{B}\left(K_{A}\right), \\
\frac{\delta S}{\delta A^{I}} & =\mathcal{B}\left(\tilde{F}_{I}\right)+\left(d^{a}{ }_{I}^{m} B_{m}-\frac{1}{3} d^{a}{ }_{J}{ }^{m} d_{m I K} A^{J K}\right) \mathcal{B}\left(G_{a}\right)+d_{m I J} A^{J} \mathcal{B}\left(\tilde{H}^{m}\right), \\
\frac{\delta S}{\delta B_{m}} & =\mathcal{B}\left(\tilde{H}^{m}\right), \\
\frac{\delta S}{\delta C^{a}} & =\mathcal{B}\left(G_{a}\right) .
\end{aligned}
$$

\footnotetext{
${ }^{29}$ We have also ignored the identities whose rank, as differential forms, is higher than eight.
} 
This identification determines completely the field theory. For instance, the equation of motion for the electric 3 -forms $C^{a}$ must be

$$
\frac{\delta S}{\delta C^{a}}=\mathcal{D}\left(\Im \mathfrak{m} \mathcal{N}_{a b} \star G^{b}+\Re \mathfrak{e} \mathcal{N}_{a b} G^{b}\right)+d_{a I}^{m} F^{I} H_{m}-Z_{a m} \mathcal{M}^{m n} \star H_{n},
$$

etc.

Can we write an action gauge-invariant action for the electric fields $\phi^{x}, A^{I}, B_{m}$ and $C^{a}$ from which these equations of motion follow, up to duality relations? We can follow the step-by-step procedure used in ref. [13] for the 5- and 6-dimensional cases. This procedure consists in considering first an action $S^{(0)}$ containing only the gauge-invariant kinetic terms for the all the electric potentials $\phi^{x}, A^{I}, B_{m}, C^{a}$ and start adding the necessary ChernSimons terms $S^{(1)}, S^{(2)}, \ldots$ to obtain the equations of motion of all the potentials occurring in $S^{(0)}$ in order of decreasing rank: $\tilde{B}^{m}, C^{a}, C_{a}, B_{m}, A^{I}$. At the first step it will be necessary to introduce terms $S^{(1)}$ containing $\tilde{B}^{m}$ but no new terms containing this potential will be introduced in the following steps. At the second step we will introduce terms $S^{(2)}$ containing $C^{a}$ (but no $\tilde{B}^{m}$ ) and in the following steps we will not introduce any more terms containing it and so on and so forth.

We will not carry this procedure to the end because in eight dimensions the number of Chern-Simons terms involving just 2- and 1-form potentials is huge and its structure is very complicated. Nevertheless we are going to check that everything works as expected for the potentials of highest $\operatorname{rank} \tilde{B}^{m}, C^{a}, C_{a}$ and we are going to find that only under certain conditions the action we are looking for exists.

Our starting point is, therefore, the action

$$
\begin{aligned}
S^{(0)}=\int\left\{-\star \mathbb{1} R+\frac{1}{2} \mathcal{G}_{x y} \mathcal{D} \phi^{x} \wedge \star \mathcal{D} \phi^{y}+\frac{1}{2} \mathcal{M}_{I J} F^{I} \wedge \star F^{J}+\frac{1}{2} \mathcal{M}^{m n} H_{m} \wedge \star H_{n}\right. \\
\left.+\frac{1}{2} G^{a} \wedge R_{a}-\star \mathbb{1} V(\phi)\right\},
\end{aligned}
$$

where we have added a scalar potential $V(\phi)$. This action gives

$$
\frac{\delta S^{(0)}}{\delta \tilde{B}^{m}}=-Z_{m}^{a} R_{a}
$$

This equations should be trivial on-duality-shell and, therefore, we must add to the action $S^{(0)}$

$$
S^{(1)}=\int Z_{m}^{a}\left(G_{a}+\frac{1}{2} Z_{a n} \tilde{B}^{n}\right) \tilde{B}^{m}
$$

so that

$$
\frac{\delta\left(S^{(0)}+S^{(1)}\right)}{\delta \tilde{B}^{m}}=-Z_{m}^{a}\left(R_{a}-G_{a}\right)
$$

The equation for $C^{a}$ that follows from $S^{(0)}+S^{(1)}$ is

$$
\frac{\delta\left(S^{(0)}+S^{(1)}\right)}{\delta C^{a}}=-\mathcal{D} R_{a}-Z_{a m} \mathcal{M}^{m n} \star H_{n},
$$


and, comparing with eq. (4.17), we see that the term $+d_{a I}{ }^{m} F^{I} H_{m}$ is missing and we must add a term of the form

$$
S^{(2)}=\int d_{a I}^{m} F^{I}\left(H_{m}-\frac{1}{2} Z_{b m} C^{b}\right) C^{a},
$$

Observe that $\tilde{B}^{m}$ does not appear in this term and its equation of motion is, therefore, not modified by it. However in this term or in any other similar term the only part of $d_{a I}^{m} Z_{b m}$ that can occur is the antisymmetric one $d_{[a \mid I}{ }^{m} Z_{\mid b] m}$ while in the term $+d_{a I}{ }^{m} F^{I} H_{m}$ both the antisymmetric and the symmetric parts occur. The only way in which we can get that term in the equations of motion is by requiring

$$
d_{(a \mid I}^{m} Z_{\mid b) m}=-\frac{1}{2} X_{I a b}=0 .
$$

Under this assumption, which will also prove crucial to obtain the equations of motion of other fields, the equation of motion of $C^{a}$ is eq. (4.17), as we wanted.

The equation of the magnetic potential $C_{a}$, which should be trivial on-duality-shell which follows from the action we have constructed is

$$
\frac{\delta\left(S^{(0)}+S^{(1)}+S^{(2)}\right)}{\delta C^{a}}=Z_{m}^{a}\left[\mathcal{M}^{m n} \star H_{n}-\mathcal{D} \tilde{B}^{m}+d_{b I}{ }^{m} F^{I} C^{b}\right] .
$$

The last two terms belong to the field strength $\tilde{H}^{m}$ and we need to add

$$
S^{(3)}=\int\left\{-\frac{1}{2} d^{b}{ }^{m} Z^{a}{ }_{m} F^{I} C_{b} C_{a}-\left[2 d^{m n p} B_{n}\left(H_{p}-2 Z_{i p} C^{i}-\frac{1}{2} \mathcal{D} B_{p}\right)+\square \tilde{H}^{m}\right] Z^{a}{ }_{m} C_{a}\right\},
$$

where $\square \tilde{H}^{m}$ is the part of the field strength $\tilde{H}^{m}$ that only contains 1-form potentials and their exterior derivatives. Observe that neither $\tilde{B}^{m}$ nor $C^{a}$ appear in this term and, therefore, their equations of motion are not modified. Observe also that we are facing here the same problem we faced in getting the equation of motion of $C^{a}$ : only $d^{[b \mid} I^{m} Z^{\mid a]} m$ can enter the action while the equation of motion contains also the symmetric part. The solution to this problem is the same: we demand

$$
d^{(a \mid}{ }_{I}^{m} Z_{m}^{\mid b)}=-\frac{1}{2} X_{I}^{a b}=0 .
$$

Using eqs. (4.24) and (4.27) The equation of motion of $B_{m}$ that follows from the action $S^{(0)}+\cdots+S^{(3)}$ can be put in the form

$$
\begin{aligned}
\frac{\delta\left(S^{(0)}+\cdots+S^{(3)}\right)}{\delta B_{m}}=- & {\left[\mathcal{D}\left(\mathcal{M}^{m n} \star H_{n}\right)+d_{a I}{ }^{m} F^{I} G^{a}-d^{a}{ }_{I}^{m} F^{I} R_{a}\right.} \\
& \left.-d^{m n p} H_{n p}-Z^{I m} \mathcal{M}_{I J} \star F^{J}\right]-d^{m n p} B_{n} Z^{a}{ }_{p}\left(R_{a}-G_{a}\right) \\
& -d^{m n p} Z^{a}{ }_{p} d_{a I}{ }^{q} B_{n} B_{q}\left(F^{I}-\frac{1}{2} Z^{I r} B_{r}\right) \\
& +d_{a I}{ }^{m} d^{a}{ }_{J}^{n} F^{I}\left(F^{I}-\frac{1}{2} Z^{I p} B_{p}\right) B_{n} \\
& +d_{a I}{ }^{m} F^{I} \square G^{a}+d^{a}{ }^{[m \mid} Z^{I \mid n]} B_{n} \square G_{a} \\
& -d^{m n p}\left(H_{n}-Z_{\text {in }} C^{i}\right)\left(H_{p}-Z_{j p} C^{j}\right) .
\end{aligned}
$$


The expression in brackets in the r.h.s. is identical to $\mathcal{B}\left(\tilde{H}^{m}\right)$ up to dualities and up to the term $d^{m}{ }_{I J K} F^{I J K}$. The next term vanishes on-duality-shell and the remaining terms should be eliminated. Observe that in the terms that need to be eliminated and introduced neither $\tilde{B}^{m}$ nor $C^{i}$ occur (they only depend on $B_{m}, A^{I}$ and their derivatives) and, therefore, their equations of motion will not be modified.

\section{Conclusions}

Following the same procedure as in refs. [7, 13], in this paper we have constructed the most general 8-dimensional theory with gauge symnmetries and with at most two derivatives: field strengths (up to 6-forms), all the Bianchi identities and duality relations satisfied by all the field strengths (up to the 9 -forms ${ }^{30}$ ), and the equations of motion of the fundamental fields. We have shown that they are characterized by a small number of invariant tensors ( $d$-tensor, embedding tensor $\vartheta$ and massive deformations $Z$ ) that satisfy certain constraints that relate them among themselves and to the structure constants and generators of the global symmetry group, which has to act on the $n_{3} 3$-form potentials of the theory as a subgroup of $\operatorname{Sp}\left(2 n_{3}, \mathbb{R}\right)$.

We have found that the Bianchi identities satisfied by the 7 -form field strengths (dual to the generalized Noether-Gaillard-Zumino current) have the general form predicted in ref. [28], although in this case it is very difficult to find the explicit form of the 7-form field strengths.

We have constructed an action from which one can derive all the equations of motion except for those of the 1-form potentials because identifying the terms that only contain 1-forms becomes extremely complicated and time-consuming.

This general result can be applied to any 8-dimensional theory with a given field content, $d$-tensors defining Chern-Simons interactions and global symmetry group, such as maximal $d=8$ supergravity.

\section{Acknowledgments}

This work has been supported in part by the Spanish Ministry of Science and Education grant FPA2012-35043-C02-01, the Centro de Excelencia Severo Ochoa Program grant SEV2012-0249, and the Spanish Consolider-Ingenio 2010 program CPAN CSD2007-00042. The work of OLA was further supported by a scholarship of the Ecuadorian Secretary of Science, Technology and Innovation. TO wishes to thank M.M. Fernández for her permanent support.

\footnotetext{
${ }^{30}$ These identities are, of course, just formal, but they encode the gauge transformations of the 8-form potentials.
} 
A Summary of relations for the generic ungauged, massless Abelian $d=$ 8 theory

\section{A.1 Field strengths}

$$
\begin{aligned}
F^{I}= & d A^{I} . \\
H_{m}= & d B_{m}-d_{m I J} F^{I} A^{J}, \\
G^{i}= & d C^{i}+d^{i}{ }_{I}^{m} F^{I} B_{m}-\frac{1}{3} d^{i}{ }_{I}^{m} d_{m J K} A^{I} F^{J} A^{K}, \\
\tilde{H}^{m}= & d \tilde{B}^{m}+d_{I}^{i}{ }_{I}^{m} C_{i} F^{I}+d^{m n p} B_{n}\left(H_{p}+\Delta H_{p}\right)+\frac{1}{12} d_{I}^{i}{ }_{I}^{m} d_{i J}{ }^{n} A^{I J} \Delta H_{n}, \\
\tilde{F}_{I}= & d \tilde{A}_{I}+2 d_{m I J} A^{J}\left(\tilde{H}_{m}-\frac{1}{2} \Delta \tilde{H}_{m}\right)-\left(d^{i}{ }_{I}^{m} B_{m}-\frac{1}{3} d^{i} J^{m} d_{m I K} A^{J K}\right)\left(G_{i}-\frac{1}{2} \Delta G_{i}\right) \\
& -\frac{1}{3}\left(d^{i} I^{m} d_{m J K}-d^{i} K^{m} d_{m I J}\right) F^{J} A^{K} C_{i}-d^{m n p} d_{m I J} A^{J} B_{n} H_{p} \\
& +\frac{1}{24}\left(d^{i} K^{m} d_{i L}{ }^{n} d_{m I J}+2 d^{i}{ }_{[I \mid}{ }^{m} d_{i \mid K]^{n}} d_{m J L}\right) F^{J} A^{K L} B_{n}+\frac{1}{24} d^{i} J^{m} d_{i K}{ }^{n} d_{m I L} A^{J K L} d B_{n} \\
& -\frac{1}{180} d^{i}{ }_{L}^{n} d_{i Q}{ }^{m} d_{m I J} d_{n P K} A^{J K L Q} F^{P},
\end{aligned}
$$

\section{A.2 Bianchi identities}

$$
\begin{aligned}
d F^{I} & =0, \\
d H_{m} & =-d_{m I J} F^{I J}, \\
d G^{i} & =d^{i}{ }_{I}^{m} F^{I} H_{m}, \\
d \tilde{H}^{m} & =d^{i}{ }_{I}{ }^{m} G_{i} F^{I}+d^{m n p} H_{n p}, \\
d \tilde{F}_{I} & =2 d_{m I J} F^{J} \tilde{H}^{m}+d_{i I}{ }^{m} G^{i} H_{m}, \\
d K_{A} & =T_{A}{ }^{I}{ }_{J} F^{J} \tilde{F}_{I}+T_{A}{ }_{n}{ }_{n} \tilde{H}^{n} H_{m}-\frac{1}{2} T_{A i j} G^{i j} .
\end{aligned}
$$

\section{A.3 Duality relations}

$$
\begin{aligned}
\star G^{i} & =\Omega^{i j} \mathcal{W}_{j k} G^{k}, \quad \text { or } \quad G_{a}^{+}=-\mathcal{N}_{a b}^{*} G^{b+}, \\
\star \tilde{H}^{m} & =\mathcal{M}^{m n} H_{n}, \\
\star \tilde{F}_{I} & =-\mathcal{M}_{I J} F^{J}, \\
\star K_{A} & =-j_{A}^{(\sigma)}, \\
\star L_{\sharp} & =-\frac{\partial V}{\partial c^{\sharp}}
\end{aligned}
$$




\section{B Summary of relations for the gauged theory}

\section{B.1 Field strengths}

$$
\begin{aligned}
F^{I}= & d A^{I}-\frac{1}{2} X_{J}{ }^{I}{ }_{K} A^{J K}+Z^{I m} B_{m} \\
H_{m}= & \mathcal{D} B_{m}-d_{m I J} d A^{I} A^{J}+\frac{1}{3} d_{m M I} X_{J}{ }^{M}{ }_{K} A^{I J K}+Z_{i m} C^{i} \\
G^{i}= & \mathcal{D} C^{i}+d^{i}{ }^{n}\left[F^{I} B_{n}-\frac{1}{2} Z^{I p} B_{n} B_{p}+\frac{1}{3} d_{n J K} d A^{J} A^{K I}\right. \\
& \left.\quad+\frac{1}{12} d_{n M J} X_{K}{ }^{M}{ }_{L} A^{I J K L}\right]-Z^{i}{ }_{m} \tilde{H}^{m} \\
\tilde{H}^{m}= & \mathcal{D} \tilde{B}^{m}-d_{i I}{ }^{m} F^{I} C^{i}+d^{m n p} B_{n}\left(H_{p}+\Delta H_{p}-2 Z_{i p} C^{i}\right) \\
& +d^{m}{ }_{I J K} d A^{I} d A^{J} A^{K} \\
& +\left(\frac{1}{12} d_{i J}{ }^{m} d^{i} K^{n} d_{n I L}-\frac{3}{4} d^{m}{ }_{I J M} X_{K}{ }^{M}{ }_{L}\right) d A^{I} A^{J K L} \\
& +\left(\frac{3}{20} d^{m}{ }_{N P M} X_{I}{ }^{N}{ }_{J}-\frac{1}{60} d_{i M}{ }^{m} d_{I}^{i n} d_{n P J}\right) X_{K}{ }^{P}{ }_{L} A^{I J K L M} \\
& +Z^{I m} \tilde{A}_{I},
\end{aligned}
$$

\section{B.2 Bianchi identities}

The Bianchi identities satisfied by the field strengths of the gauged theory are $\mathcal{B}(\cdot)=0$ where

$$
\begin{aligned}
\mathcal{B}\left(L_{A}{ }^{I}\right) & =-\left[\mathcal{D} L_{A}{ }^{I}+F^{I} K_{A}+W^{I} A^{\beta} M_{\beta}\right], \\
\mathcal{B}\left(L_{I m}\right) & =-\left[\mathcal{D} L_{I m}+\tilde{F}_{I} H_{m}+W_{I m}{ }^{\beta} M_{\beta}\right], \\
\mathcal{B}\left(L^{i m}\right) & =-\left[\mathcal{D} L^{i m}+G^{i} \tilde{H}^{m}+W^{i m} \beta M_{\beta}\right], \\
\mathcal{B}\left(K_{A}\right) & =\mathcal{D} K_{A}-T_{A}{ }^{I}{ }_{J} F^{J} \tilde{F}_{I}-T_{A}{ }^{m}{ }_{n} \tilde{H}^{n} H_{m}+\frac{1}{2} T_{A i j} G^{i j}-Y_{A}^{\sharp} L_{\sharp}, \\
\mathcal{B}\left(\tilde{F}_{I}\right) & =-\left[\mathcal{D} \tilde{F}_{I}-2 d_{m I J} F^{J} \tilde{H}^{m}-d_{i I}{ }^{m} G^{i} H_{m}+2 d^{m}{ }_{I J K} F^{J K} H_{m}+\vartheta_{I}{ }^{A} K_{A}\right], \\
\mathcal{B}\left(\tilde{H}^{m}\right) & =-\left[\mathcal{D} \tilde{H}^{m}+d_{i I}{ }^{m} F^{I} G^{i}-d^{m n p} H_{n p}-d^{m}{ }_{I J K} F^{I J K}-Z^{I m} \tilde{F}_{I}\right], \\
\mathcal{B}\left(G_{i}\right) & =-\left[\mathcal{D} G_{i}-d_{i I}{ }^{m} F^{I} H_{m}+Z_{i m} \tilde{H}^{m}\right], \\
\mathcal{B}\left(H_{m}\right) & =-\left[\mathcal{D} H_{m}+d_{m I J} F^{I J}-Z_{i m} G^{i}\right], \\
\mathcal{B}\left(F^{I}\right) & =-\left[\mathcal{D} F^{I}-Z^{I m} H_{m}\right], \\
\mathcal{B}(\mathcal{D} \mathcal{M}) & =-\left[\mathcal{D} \mathcal{D} \mathcal{M}+F^{I} \vartheta_{I}^{A} \delta_{A} \mathcal{M}\right] \\
\mathcal{B}\left(c^{\sharp}\right) & =\mathcal{D} c^{\sharp} \\
\mathcal{B}\left(\mathcal{Q}^{\beta}\right) & =\mathcal{Q}{ }^{\beta} .
\end{aligned}
$$

Here $\sharp$ labels the deformation parameters and $\beta$ the constraints, as discussed in sections 3.7 and 3.8 . 


\section{B.3 Identities of Bianchi identities}

$$
\begin{aligned}
\mathcal{D B}\left(F^{I}\right)-Z^{I m} \mathcal{B}\left(H_{m}\right) & =0, \\
\mathcal{D} \mathcal{B}\left(H_{m}\right)-2 d_{m I J} F^{I} \mathcal{B}\left(F^{J}\right)+Z_{i m} G^{i} & =0, \\
\mathcal{D B}\left(G_{i}\right)-d_{i I}{ }^{m}\left[\mathcal{B}\left(H_{m}\right) F^{I}+H_{m} \mathcal{B}\left(F^{I}\right)\right]-Z_{i m} \mathcal{B}\left(\tilde{H}^{m}\right) & =0, \\
\mathcal{D B}\left(\tilde{H}^{m}\right)-d_{i I}{ }^{m}\left[\mathcal{B}\left(G^{i}\right) F^{I}+G^{i} \mathcal{B}\left(F^{I}\right)\right]+2 d^{m n p} \mathcal{B}\left(H_{n}\right) H_{p} & \\
-3 d^{m}{ }_{I J K} \mathcal{B}\left(F^{I}\right) F^{J K}+Z^{I m} \mathcal{B}\left(\tilde{F}_{I}\right) & =0, \\
\mathcal{D B}\left(\tilde{F}_{I}\right)+2 d_{m I J}\left[\mathcal{B}\left(\tilde{H}^{m}\right) F^{J}+\tilde{H}^{m} \mathcal{B}\left(F^{J}\right)\right] & \\
+d_{i I}{ }^{m}\left[\mathcal{B}\left(G^{i}\right) H_{m}+G^{i} \mathcal{B}\left(H_{m}\right)\right] & \\
-3 d^{m}{ }_{I J K}\left[2 \mathcal{B}\left(F^{J}\right) F^{K} H_{m}+F^{J}{ }_{\mathcal{B}}\left(H_{m}\right)\right]+\vartheta_{I}{ }^{A} \mathcal{B}\left(K_{A}\right) & =0, \\
\mathcal{D B}\left(K_{A}\right)+T_{A}{ }^{I}{ }_{J}\left[\mathcal{B}\left(F^{J}\right) \tilde{F}_{I}+F^{J} \mathcal{B}\left(\tilde{F}_{I}\right)\right] & \\
\left.\tilde{H}^{n} \mathcal{B}\left(H_{m}\right)\right]+T_{A i j}{ }^{m}{ }_{n}{ }_{n}{ }^{i} \mathcal{B}\left(G^{j}\right)+Y_{A}^{\sharp} \mathcal{B}\left(L_{\sharp}\right) & =0 .
\end{aligned}
$$

Open Access. This article is distributed under the terms of the Creative Commons Attribution License (CC-BY 4.0), which permits any use, distribution and reproduction in any medium, provided the original author(s) and source are credited.

\section{References}

[1] A. Salam and E. Sezgin, Supergravities in diverse dimensions, vol. 1 and 2, World Scientific, Singapore (1989) [INSPIRE].

[2] F. Cordaro, P. Fré, L. Gualtieri, P. Termonia and M. Trigiante, $N=8$ gaugings revisited: an exhaustive classification, Nucl. Phys. B 532 (1998) 245 [hep-th/9804056] [InSPIRE].

[3] H. Nicolai and H. Samtleben, Maximal gauged supergravity in three-dimensions, Phys. Rev. Lett. 86 (2001) 1686 [hep-th/0010076] [INSPIRE].

[4] H. Nicolai and H. Samtleben, Compact and noncompact gauged maximal supergravities in three-dimensions, JHEP 04 (2001) 022 [hep-th/0103032] [INSPIRE].

[5] B. de Wit, H. Samtleben and M. Trigiante, On Lagrangians and gaugings of maximal supergravities, Nucl. Phys. B 655 (2003) 93 [hep-th/0212239] [INSPIRE].

[6] B. de Wit, H. Samtleben and M. Trigiante, Magnetic charges in local field theory, JHEP 09 (2005) 016 [hep-th/0507289] [INSPIRE].

[7] T. Ortín, Gravity and strings, $2^{\text {nd }}$ edition, Cambridge University Press, Cambridge U.K. (2015) [INSPIRE].

[8] B. de Wit and H. Samtleben, Gauged maximal supergravities and hierarchies of non-Abelian vector-tensor systems, Fortsch. Phys. 53 (2005) 442 [hep-th/0501243] [INSPIRE].

[9] B. de Wit, H. Nicolai and H. Samtleben, Gauged supergravities, tensor hierarchies and M-theory, JHEP 02 (2008) 044 [arXiv:0801.1294] [INSPIRE].

[10] E.A. Bergshoeff, J. Hartong, O. Hohm, M. Hübscher and T. Ortín, Gauge theories, duality relations and the tensor hierarchy, JHEP 04 (2009) 123 [arXiv:0901.2054] [INSPIRE].

[11] B. de Wit and M. van Zalk, Supergravity and M-theory, Gen. Rel. Grav. 41 (2009) 757 [arXiv:0901.4519] [INSPIRE]. 
[12] E. Bergshoeff, H. Samtleben and E. Sezgin, The gaugings of maximal $D=6$ supergravity, JHEP 03 (2008) 068 [arXiv:0712.4277] [INSPIRE].

[13] J. Hartong and T. Ortín, Tensor hierarchies of 5- and 6-dimensional field theories, JHEP 09 (2009) 039 [arXiv:0906.4043] [INSPIRE].

[14] O. Hohm and Y.-N. Wang, Tensor hierarchy and generalized Cartan calculus in $\mathrm{SL}(3) \times \mathrm{SL}(2)$ exceptional field theory, JHEP 04 (2015) 050 [arXiv: 1501.01600] [INSPIRE].

[15] G. Dibitetto, J.J. Fernández-Melgarejo, D. Marqués and D. Roest, Duality orbits of non-geometric fluxes, Fortsch. Phys. 60 (2012) 1123 [arXiv:1203.6562] [INSPIRE].

[16] G. Dall'Agata, G. Inverso and A. Marrani, Symplectic deformations of gauged maximal supergravity, JHEP 07 (2014) 133 [arXiv:1405.2437] [INSPIRE].

[17] G. Dall'Agata, G. Inverso and M. Trigiante, Evidence for a family of $\mathrm{SO}(8)$ gauged supergravity theories, Phys. Rev. Lett. 109 (2012) 201301 [arXiv:1209.0760] [INSPIRE].

[18] J. Scherk and J.H. Schwarz, How to get masses from extra dimensions, Nucl. Phys. B 153 (1979) 61 [INSPIRE].

[19] A. Salam and E. Sezgin, D = 8 supergravity, Nucl. Phys. B 258 (1985) 284 [inSPIRE].

[20] N. Alonso Alberca, E. Bergshoeff, U. Gran, R. Linares, T. Ortín and D. Roest, Domain walls of $D=8$ gauged supergravities and their $D=11$ origin, JHEP 06 (2003) 038 [hep-th/0303113] [INSPIRE].

[21] E. Bergshoeff, U. Gran, R. Linares, M. Nielsen, T. Ortín and D. Roest, The Bianchi classification of maximal D =8 gauged supergravities, Class. Quant. Grav. 20 (2003) 3997 [hep-th/0306179] [INSPIRE].

[22] N. Alonso-Alberca, P. Meessen and T. Ortín, An SL(3,Z) multiplet of eight-dimensional type-II supergravity theories and the gauged supergravity inside, Nucl. Phys. B 602 (2001) 329 [hep-th/0012032] [INSPIRE].

[23] P. Meessen and T. Ortín, An $\mathrm{SL}(2, Z)$ multiplet of nine-dimensional type-II supergravity theories, Nucl. Phys. B 541 (1999) 195 [hep-th/9806120] [INSPIRE].

[24] J. Gheerardyn and P. Meessen, Supersymmetry of massive D $=9$ supergravity, Phys. Lett. B 525 (2002) 322 [hep-th/0111130] [INSPIRE].

[25] N. Alonso-Alberca and T. Ortín, Gauged/massive supergravities in diverse dimensions, Nucl. Phys. B 651 (2003) 263 [hep-th/0210011] [INSPIRE].

[26] Ó. Lasso Andino and T. Ortín, A 1-parameter family of $\mathrm{SO}(3)$-gauged maximal $D=8$ supergravities, arXiv:1605.09629 [INSPIRE].

[27] L. Andrianopoli et al., $N=2$ supergravity and $N=2$ super Yang-Mills theory on general scalar manifolds: symplectic covariance, gaugings and the momentum map, J. Geom. Phys. 23 (1997) 111 [hep-th/9605032] [INSPIRE].

[28] I.A. Bandos and T. Ortín, On the dualization of scalars into $(d-2)$-forms in supergravity. Momentum maps, R-symmetry and gauged supergravity, JHEP 08 (2016) 135 [arXiv: 1605.05559] [INSPIRE].

[29] M.K. Gaillard and B. Zumino, Duality rotations for interacting fields, Nucl. Phys. B 193 (1981) 221 [INSPIRE].

[30] E.A. Bergshoeff, J. Gomis, T.A. Nutma and D. Roest, Kac-Moody spectrum of (half-)maximal supergravities, JHEP 02 (2008) 069 [arXiv:0711.2035] [INSPIRE]. 